\title{
Null controllability of the heat equation in pseudo-cylinders by an internal control.
}

\author{
Jon Asier Bárcena-Petisco*†
}

December 26, 2019

\section{Abstract and basic information}

In this paper we prove the null controllability of the heat equation in domains with a cylindrical part and limited by a Lipschitz graph. The proof consists mainly on getting a Carleman estimate which presents the usual absorption properties. The main difficulty we face is the loss of existence of the usual weighted function in $C^{2}$ smooth domains. In order to deal with this, we use its cylindrical structure and approximate the system by the same system stated in regular domains. Finally, we show some applications like the controllability of the semi-linear heat equation in those domains.

Key words: Carleman inequalities, heat equation, Lipschitz domains, null controllability

AMS subject classification: 35D30, 93B05, 93C20

Abbreviated title: Null controllability in pseudo-cylinders

Acknowledgements: I would like to thank my thesis advisor Sergio Guerrero, two anonymous referees and Luis Escauriaza for their helpful comments and remarks, which have improved the quality of this paper. This work was supported by grants from Région Ile-de-France. This work has been partially supported by the ANR research project IFSMACS (ANR-15-CE40-0010).

\footnotetext{
*E-mail: barcena@ljll.math.upmc.fr

${ }^{\dagger}$ Sorbonne Université, Laboratoire Jacques-Louis Lions, F-75005, Paris, France
} 


\section{Introduction}

The null controllability of the heat equation has been an interesting research topic in the last 40 years. Regarding this topic, in this paper we focus on domains; that is, on bounded connected non-empty open sets of $\mathbb{R}^{d}$. There are three main approaches in the literature:

- The first one uses the observability estimates of the wave equation to prove the null controllability of the heat equation. This method dates back to 1973 (see [34]) and recently, it has been used to get accurate bounds on the cost of the null controllability (see, for instance, 31, 32, 37] and [10]).

- The second one is based on spectral inequalities and on properties of analytic functions. The first time this approach is used for the heat equation was in 1995 in [27], where the authors proved the controllability in $C^{\infty}$ domains. More recently, the null controllability of the heat equation has been studied with these techniques when the control is located in an arbitrary measurable set of positive measure (see, for instance, [38], [39] and [2]), which is one of the advantages of this method. In addition, these results have recently been extended to Lipschitz domains which are locally star-shaped (see [3]). As for other parabolic equations, this method has been used recently to study the controllability of the Stokes equation (see [6]) and higher order parabolic equations (see, for instance, [1] and [12]).

- The third one is based on multiplying the adjoint function by an exponential weight and using the PDE satisfied by the new function. Indeed, Fursikov and Imanuvilov proved with this method in 1996 the controllability of the heat equation in $C^{2}$ domains (see [19]) when the control domain is an open set. This method is really flexible and it is the base to study the controllability of countless parabolic differential equations, both linear and non-linear (see, for instance, [19]). As a continuation of the work made in [19], Carleman estimates of the heat equation have been established when the system is not regular, which proves the null controllability of some related linear and non-linear systems. For instance, in [24] and [16] the authors consider non-regular source terms. Moreover, in [15] and [23] the authors study some boundary conditions which imply that the solution is not in $L^{2}\left(0, T ; H^{2}(\Omega)\right)$. Additional examples are given in [9], 4] and [26], where the authors analyse parabolic equations in which the diffusion coefficient is not continuous. Finally, it is well-known that this method can be easily generalized to cubes or, more generally, to any cartesian product of $C^{2}$ domains (see, for instance, [20]). 
The main objective of this paper is to extend the method of Fursikov and Imanuvilov to additional domains which are not $C^{2}$ when there is a lack of regularity caused by the domain and the control acts on an internal subdomain. In particular, we aim to get a Carleman inequality with a source term. It is a relevant problem because, unlike in [3], this method allows us to consider lower order terms with coefficients that just belong to $L^{\infty}$, and hence treat nonlinearities. We recall that the authors in [19] prove a Carleman inequality with the help of an auxiliary function $\eta$ which satisfies:

$$
\eta \in C^{2}(\bar{\Omega}), \quad \eta=0 \text { on } \partial \Omega, \quad \eta>0 \text { in } \Omega, \quad \inf _{\bar{\Omega} \backslash \omega}|\nabla \eta|>0,
$$

for $\omega$ the control domain. Since in a Lipschitz domain the heat equation has a unique energy solution, the existence of some Carleman estimate seems reasonable. However, two difficulties arise:

- The first and main difficulty is that the construction of the function $\eta$ given in [19] does not work when $\Omega$ is not $C^{2}$. Indeed, if $\Omega$ has corners and if $\omega$ is compactly included in $\Omega$, the three conditions in (1.1) are incompatible. The only solution known so far for other domains (like cylinders) is to construct manually an auxiliary function which satisfies the following assumptions (which are verified by any $\eta$ satisfying (1.1)):

$$
\eta \in C^{2}(\bar{\Omega}), \quad \partial_{n} \eta \leq 0 \text { on } \partial \Omega, \quad \inf _{\bar{\Omega} \backslash \omega}|\nabla \eta|>0,
$$

for $n$ the outward unit normal vector on $\partial \Omega$. In the case of cylinders, the construction of $\eta$ is done for instance as a sum of functions of different variables.

- The second difficulty is that [19] uses that the Laplacian behaves well in $\Omega$ in the sense that $\mathcal{D}_{\Omega}(\Delta) \subset H^{2}(\Omega)$ continuously, for

$$
\mathcal{D}_{\Omega}(\Delta):=\left\{w \in H_{0}^{1}(\Omega): \Delta w \in L^{2}(\Omega)\right\}
$$

omitting the $\Omega$ if it is clear. However, when $\Omega$ is Lipschitz this is not always true, since in a general Lipschitz domain the most we can ensure is that $\mathcal{D}(\Delta) \subset H^{3 / 2}(\Omega)$ continuously. Indeed, the fact that $\mathcal{D}(\Delta) \subset H^{3 / 2}(\Omega)$ continuously is proved in [25], and for all $\varepsilon>0$ a counter-example of a domain $\Omega_{\varepsilon}$ for which there is $w \in H_{0}^{1}\left(\Omega_{\varepsilon}\right)$ such that $-\Delta w \in C^{\infty}\left(\overline{\Omega_{\varepsilon}}\right)$ but $w \notin H^{3 / 2+\varepsilon}(\Omega)$ is given for instance in [21]. In particular, if $u$ is a solution of the backwards heat equation with Dirichlet boundary conditions, initial value in $H_{0}^{1}(\Omega)$ and source term in $L^{2}((0, T) \times \Omega)$, the most that we can expect is that $u \in H^{1}\left(0, T ; L^{2}(\Omega)\right) \cap L^{2}(0, T ; \mathcal{D}(\Delta))$. However, while doing the Carleman in [19] the authors do some calculations with the second order derivatives, so they need that the regular solutions of the heat equation belong to $L^{2}\left(0, T ; H^{2}(\Omega)\right)$. One of the contributions of 
this paper on the Fursikov-Imanuvilov method is reducing the difficulty that the $L^{2}$-norm of the Laplacian is not equivalent to the $H^{2}$-norm to the existence of a proper auxiliary function. We do it in Section 2 by approximating the domain with regular subdomains.

As a first step to prove the null controllability of the heat equation by an internal control in any Lipschitz domain, in this paper we prove the null controllability in pseudo-cylinders. By pseudo-cylinder we mean the following:

Definition 1.1. We say that $\Omega \subset \mathbb{R}^{d+1}(d \geq 1)$ is a pseudo-cylinder if there are $\mathfrak{B} \subset \mathbb{R}^{d}$ a $C^{2}$ domain, $U$ the composition of a rotation and a translation and $H: \overline{\mathfrak{B}} \mapsto \mathbb{R}^{+}$a Lipschitz function satisfying $\inf _{\mathfrak{B}} H>0$ such that:

$$
\Omega=U(\{(x, z): x \in \mathfrak{B}, z \in(0, H(x))\})
$$

Moreover, we denote the cylindrical part by:

$$
\mathfrak{C}:=U\left(\mathfrak{B} \times\left(0, \inf _{\mathfrak{B}} H\right)\right) .
$$

If $U=I$ (the identity endomorphism), we say that the cylinder is canonically oriented. In that case, we have:

$$
\mathfrak{C}=\mathfrak{B} \times\left(0, \inf _{\mathfrak{B}} H\right)
$$

Remark 1.2. If $\Omega$ is a pseudo-cylinder, we can split $\partial \Omega$ into three parts:

$$
U(\mathfrak{B} \times\{0\}), \quad \mathfrak{L}:=U(\{(x, z): x \in \partial \mathfrak{B}, z \in(0, H(x))\}), \quad \mathfrak{T}:=U(\{(x, H(x)): x \in \mathfrak{B}\})
$$

If $\Omega$ is canonically oriented, those parts are given by:

$$
\mathfrak{B} \times\{0\}, \quad \mathfrak{L}=\{(x, z): x \in \partial \mathfrak{B}, z \in(0, H(x))\}, \quad \mathfrak{T}=\{(x, H(x)): x \in \mathfrak{B}\} .
$$

Example 1.3. See Figure 1 for a canonically-oriented pseudo-cylinder in $\mathbb{R}^{2}$.

Remark 1.4. The pseudo-cylinders are relevant domains, for example, in fluid mechanics (see, for instance, [33] and [22]).

In this paper we solve the previously presented difficulties for pseudo-cylinders and get some Carleman estimates (see Proposition 3.2 and Proposition 3.6 below) which present the usual absorption properties. However, as we explain in Comment 5 of Section 3.3 , our method does not work in every Lipschitz domain, so the general case remains an interesting open problem. 


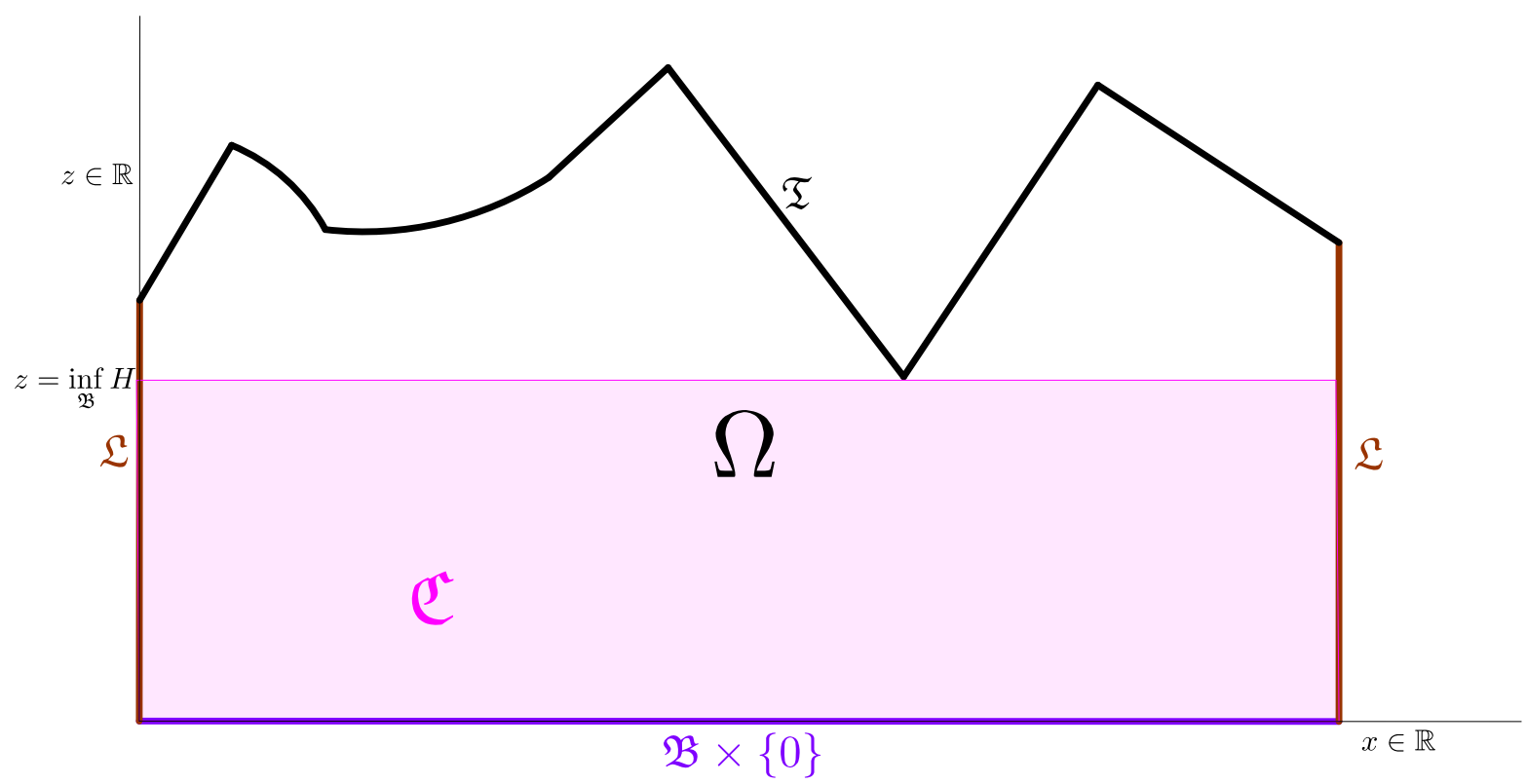

Figure 1: A canonically oriented pseudo-cylinder.

Before presenting the main result of this paper we introduce the following notation:

Definition 1.5. Let $\Omega$ be any domain and let $\omega \subset \Omega$ be a subdomain. We define as usual $Q:=(0, T) \times \Omega, Q_{\omega}:=(0, T) \times \omega, \Sigma:=(0, T) \times \partial \Omega$ and $n$ the outward unit normal vector on $\partial \Omega$. Similarly, if the domain is denoted by $\tilde{\Omega}$, we define $\tilde{Q}:=(0, T) \times \tilde{\Omega}, \tilde{\Sigma}:=(0, T) \times \partial \tilde{\Omega}$ and $\tilde{n}$ as the outward unit normal vector on $\partial \tilde{\Omega}$. Moreover, we denote by $\omega \subset \subset \Omega$ if $\omega$ is compactly included in $\Omega$. Finally, in order to shorten the notation we omit the "dt", "dx" and "dz" when we are integrating and the integrated variables can be easily deduced by looking at the integration domain.

Now, we may state the main result of this paper:

Theorem 1.6. Let $\Omega$ be a pseudo-cylinder and $\omega \subset \Omega$ be a subdomain. Then, there is $C>0$ such that if $T>0, A \in\left(L^{\infty}(Q)\right)^{d+1}, a \in L^{\infty}(Q)$, and $y^{0} \in L^{2}(\Omega)$, there is a control $v \in L^{2}\left(Q_{\omega}\right)$ such that the solution of the system:

$$
\begin{cases}y_{t}-\Delta y+A \cdot \nabla y+a y=v 1_{\omega} & \text { in } Q \\ y=0 & \text { on } \Sigma, \\ y(0, \cdot)=y^{0} & \text { on } \Omega,\end{cases}
$$

satisfies $y(T, \cdot)=0$, and such that the control satisfies the estimate:

$$
\|v\|_{L^{2}\left(Q_{\omega}\right)} \leq C e^{C K(T, a, A)}\left\|y^{0}\right\|_{L^{2}(\Omega)},
$$


for:

$$
K(T, a, A):=1+T^{-1}+T\|a\|_{L^{\infty}(Q)}+\|a\|_{L^{\infty}(Q)}^{2 / 3}+(1+T)\|A\|_{\left(L^{\infty}(Q)\right)^{d+1}}^{2} .
$$

Remark 1.7. Since the Laplacian is invariant under rotations (and translations), it suffices to prove Theorem 1.6 when $\Omega$ is canonically oriented. Moreover, we remark that if we prove Theorem 1.6 for some control domain $\tilde{\omega}$, the conclusions of Theorem 1.6 are true for all $\omega \supset \tilde{\omega}$, as it suffices to consider $v=\tilde{v} 1_{\tilde{\omega}}$, for $\tilde{v}$ the control supported in $\tilde{\omega}$.

Using Theorem 1.6 we can get positive results about the controllability of the semi-linear heat equation when the non-linearity depends only on $y$ (see Comment 6 on Section 3.3 for a remark about non-linearities depending on $\nabla y$ ):

$$
\begin{cases}y_{t}-\Delta y+f(y)=v 1_{\omega} & \text { in } Q, \\ y=0 & \text { on } \Sigma, \\ y(0, \cdot)=y^{0} & \text { on } \Omega .\end{cases}
$$

Indeed, under weak non-linearities we have controllability to trajectories and approximate controllability:

Corollary 1.8. Let $\Omega$ be a pseudo-cylinder, $\omega \subset \Omega$ be a subdomain, $T>0$ and $y^{*} \in C\left([0, T] ; L^{2}(\Omega)\right)$ be a solution of (1.6) corresponding to some data $\left(y^{0}\right)^{*} \in L^{2}(\Omega)$ and $v^{*} \in L^{\infty}\left(Q_{\omega}\right)$. Assume that $f: \mathbb{R} \mapsto \mathbb{R}$ is locally Lipschitz and satisfies almost everywhere in $\mathbb{R}$ :

$$
\left|f^{\prime}(s)\right| \leq C\left(1+|s|^{1+4 / d}\right),
$$

and:

$$
\lim _{|s| \rightarrow \infty} \frac{f(s)}{|s| \log ^{3 / 2}(1+|s|)}=0 .
$$

Then, for all $y^{0} \in L^{2}(\Omega)$ there is $v \in L^{\infty}\left(Q_{\omega}\right)$ such that the solution of 1.6 belongs to $C\left([0, T] ; L^{2}(\Omega)\right)$ and satisfies $y(T, \cdot)=y^{*}(T, \cdot)$.

Corollary 1.9. Let $\Omega$ be a pseudo-cylinder, $\omega \subset \Omega$ be a domain, $T>0$ and let us assume that (1.6) has a solution $y^{*} \in C\left([0, T] ; L^{2}(\Omega)\right)$ corresponding to some initial state in $\left(y^{0}\right)^{*} \in L^{2}(\Omega)$ and to some control function $v^{*} \in L^{\infty}\left(Q_{\omega}\right)$. Assume that $f$ is locally Lipschitz and satisfies (1.7) almost everywhere in $\mathbb{R}$ and 1.8 . Then, for all $y^{1} \in L^{2}(\Omega)$ and all $\varepsilon>0$ there is $v \in L^{\infty}\left(Q_{\omega}\right)$ such that 1.6$]$ has a solution in $C\left([0, T] ; L^{2}(\Omega)\right)$ satisfying:

$$
\left\|y(T, \cdot)-y^{1}\right\| \leq \varepsilon .
$$

In order to see that Theorem 1.6 implies Corollaries 1.8 and 1.9 it suffices to follow step by step the proof given in [18] for the case in which $\Omega$ is a $C^{2}$ domain. Indeed, in order to 
see that results on Sobolev spaces used in [18] remain true when $\Omega$ is just Lipschitz, the reader can consult for instance [1], [5] and [36]. In addition, we can find in [18] some facts about controllability of the semi-linear heat equation which remain true in pseudo-cylinders, like that 1.6 is not approximately controllable for $f(s)=\int_{0}^{s} \log ^{p}(1+\sigma) d \sigma, p>2$ and $\omega \subset \subset \Omega$.

Remark 1.10. The results presented in this paper differ from [3] because of the following reasons:

- There are some pseudo-cylinders which are not locally star-shaped (see Appendix B); thus, this is the first time that the null controllability of the heat equation is proved in these domains. In addition, there are locally star-shaped domains which are not pseudo-cylinders (for example, a disk or a pentagon). Of course, there also are pseudo-cylinders which are locally star-shaped (for instance, the rectangle).

- The coefficient of the lower order terms in Theorem 1.6 can depend on $t$ and $x$, whereas in [3] the coefficients only depend on $t$.

- Thanks to the fact that we can establish controllability results for the heat equation with coefficients that depend on $t$ and $x$, we can prove controllability results for the semi-linear heat equation, which is an open problem of [3].

- In [3] the control is located in an arbitrary measurable subset of strictly positive measure, whereas in this paper the control is located in open subsets.

In this document we consider the following weights defined in $Q$ :

$$
\alpha_{\eta}:=\frac{e^{\lambda \frac{2 m+1}{2 m} k}-e^{\lambda(k+\eta)}}{t^{m}(T-t)^{m}}, \quad \xi_{\eta}:=\frac{e^{\lambda(k+\eta)}}{t^{m}(T-t)^{m}},
$$

for $m \geq 1$ a real constant, $\eta$ some auxiliary $C^{2}(\bar{\Omega})$ function (which, unlike it is usually done in the literature, may take negative values) and $k>2(m+1)\|\eta\|_{L^{\infty}(\Omega)}$ a real constant, all of them to be fixed later. In (1.9) the subindex $\eta$ might be omitted if it is clear. Moreover, if we denote some function by $\tilde{\eta}$, we use the notation $\tilde{\alpha}$ and $\tilde{\xi}$ to refer to $\alpha_{\tilde{\eta}}$ and $\xi_{\tilde{\eta}}$, respectively. We recall that we have the following estimates for the weights given in $(1.9)$, for some $C>0$ that is universal (independent of any variable like $\Omega, \eta, m, k, s, \lambda, \ldots$ ):

$$
\left|\xi_{t}\right|+\left|\alpha_{t}\right| \leq C m T \xi^{(m+1) / m}, \quad\left|\alpha_{t t}\right| \leq C\left(m \xi^{(m+1) / m}+m(m+1) T^{2} \xi^{(m+2) / m}\right) .
$$

Weights similar to 1.9 were first introduced in [19]. However, our version is more similar to the one presented in [17], though we have done slight modifications to also consider the situations in which $\eta$ is not a positive function. 
Remark 1.11. We need $k>2(m+1)\|\eta\|_{L^{\infty}(\Omega)}$ to assure that the numerator of $\alpha$ is strictly positive, so that we can integrate by parts in the time variable with a null boundary term when proving the Carleman inequality. Moreover, we also need it to prove (1.10).

Remark 1.12. These weights, stated as in (1.9), have the advantage that can be compared easily when using two different auxiliary functions. In particular, if we have $\eta_{1}$ and $\eta_{2}$ defined in a canonically oriented pseudo-cylinder, for any $m \geq 1, k>2(m+1) \max \left\{\left\|\eta_{1}\right\|_{L^{\infty}(\Omega)},\left\|\eta_{2}\right\|_{L^{\infty}(\Omega)}\right\}$, $t \in(0, T)$ and $(x, z) \in \bar{\Omega}$, we find:

$$
\xi_{\eta_{1}}(t, x, z) \leq \xi_{\eta_{2}}(t, x, z) \text { if and only if } \eta_{1}(x, z) \leq \eta_{2}(x, z)
$$

and:

$$
-\alpha_{\eta_{1}}(t, x, z) \leq-\alpha_{\eta_{2}}(t, x, z) \text { if and only if } \eta_{1}(x, z) \leq \eta_{2}(x, z)
$$

Let us explain briefly how to solve the two problems presented at the beginning of this section on pseudo-cylinders:

- Concerning the construction of the auxiliary function, which is the main difficulty, we consider different approaches depending on the control domain. If $\omega \subset \Omega$ we use an approach consisting of expressing $\eta$ as a sum of two different variables, whereas if $\omega \subset \Omega \backslash \mathfrak{C}$, we "transmit" the estimate from one control domain to another with a second auxiliary function. This is done in Section 3 .

- As for dealing with the fact that $\mathcal{D}(\Omega) \not \subset H^{2}(\Omega)$, we just have to approximate $\Omega$ by $C^{2}$ domains which are compatible with $\eta$ (once $\eta$ is constructed). This is explained in Section 2 for Lipschitz domains.

The rest of the paper is organised as follows: in Section 2 we explain how to deal with $\Omega$ being just Lipschitz, in Section 3 we get the Carleman inequalities, in Appendix $\mathrm{A}$ we prove the compatibility of the proposed auxiliary function with the domain and in Appendix B we show that there are pseudo-cylinders which are not locally star-shaped.

\section{A Carleman inequality for some Lipschitz domains}

Let $\Omega$ be a Lipschitz domain. We define:

$$
\mathcal{W}_{\Omega}:=L^{2}\left(0, T ; H_{0}^{1}(\Omega)\right) \cap H^{1}\left(0, T ; H^{-1}(\Omega)\right),
$$

omitting the subindex $\Omega$ if it is clear. In addition, we define: 
Definition 2.1. Let $\Omega$ be a Lipschitz domain, let $u^{0} \in L^{2}(\Omega)$ and let $g \in L^{2}(Q)$. Then, we denote by:

$$
\mathbb{S}\left(\Omega, u^{0}, g\right)(t, x)
$$

the only solution in $\mathcal{W}$ of:

$$
\begin{cases}u_{t}-\Delta u=g & \text { in } Q, \\ u=0 & \text { on } \Sigma, \\ u(0, \cdot)=u^{0} & \text { on } \Omega .\end{cases}
$$

Finally, we define the convergence of domains in the following way:

Definition 2.2. Let $\varepsilon_{0}>0, \Omega$ be a domain and $\left(\Omega_{\varepsilon}\right)_{\varepsilon \in\left(0, \varepsilon_{0}\right)}$ be domains such that $\Omega_{\varepsilon} \subset \Omega$ for all $\varepsilon \in\left(0, \varepsilon_{0}\right)$. We say that $\Omega_{\varepsilon} \rightarrow_{d} \Omega$ if:

$$
\lim _{\varepsilon \rightarrow 0^{+}} \sup _{\Omega \backslash \Omega_{\varepsilon}} d(\cdot, \partial \Omega)=0
$$

Remark 2.3. If $\Omega_{\varepsilon} \rightarrow_{d} \Omega$, then $\Omega_{\varepsilon}$ converges to $\Omega$ in measure; that is, $\left\|1_{\Omega \backslash \Omega_{\varepsilon}}\right\|_{L^{1}\left(\mathbb{R}^{d}\right)} \rightarrow 0$, because of the Dominated Convergence Theorem.

We have the following technical result:

Lemma 2.4. Let $\Omega$ be a Lipschitz domain, $\Omega_{\varepsilon} \subset \Omega$ be some Lipschitz domains such that:

$$
\Omega_{\varepsilon} \rightarrow_{d} \Omega
$$

$u^{0} \in L^{2}(\Omega)$ and $g \in L^{2}(Q)$. Then,



As far as we know, the proof of Lemma 2.4 is not available in the literature, so we give a proof, whose originality we do not claim. For the proof of Lemma 2.4 we need the following interpolation result, proved for instance in [30] and [29]:

Lemma 2.5. Let $\Omega$ be a Lipschitz domain. Then,

1. The injection $\mathcal{W} \subset L^{2}(Q)$ is compact.

2. The injection $\mathcal{W} \subset C\left([0, T] ; L^{2}(\Omega)\right)$ is continuous.

3. For all $u, w \in \mathcal{W}$, we have $\langle u(t, \cdot), w(t, \cdot)\rangle_{L^{2}(\Omega)} \in W^{1,1}(0, T)$ and:

$$
\partial_{t}\left(\langle u(t, \cdot), w(t, \cdot)\rangle_{L^{2}(\Omega)}\right)=\left\langle\partial_{t} u(t, \cdot), w(t, \cdot)\right\rangle_{H^{-1}(\Omega) \times H_{0}^{1}(\Omega)}+\left\langle\partial_{t} w(t, \cdot), u(t, \cdot)\right\rangle_{H^{-1}(\Omega) \times H_{0}^{1}(\Omega)} .
$$


Proof of Lemma 2.4. In order to prove Lemma 2.4 we recall that for any Lipschitz domain $\tilde{\Omega}$, any $\tilde{u}^{0} \in L^{2}(\tilde{\Omega})$ and any $\tilde{g} \in L^{2}(Q), \mathbb{S}\left(\tilde{\Omega}, \tilde{u}^{0}, \tilde{g}\right)$ is characterized as the only element in $\mathcal{W}_{\tilde{\Omega}}$ such that:

$$
\left\{\begin{array}{l}
\int_{0}^{T}\left\langle\partial_{t} \mathbb{S}\left(\tilde{\Omega}, \tilde{u}^{0}, \tilde{g}\right), \phi\right\rangle_{H^{-1}(\tilde{\Omega}), H_{0}^{1}(\tilde{\Omega})}+\iint_{\tilde{Q}} \nabla \mathbb{S}\left(\tilde{\Omega}, \tilde{u}^{0}, \tilde{g}\right) \cdot \nabla \phi=\iint_{\tilde{Q}} g \phi \quad \forall \phi \in \mathcal{D}(\tilde{Q}) \\
\mathbb{S}\left(\tilde{\Omega}, \tilde{u}^{0}, \tilde{g}\right)(0, \cdot)=\tilde{u}^{0}
\end{array}\right.
$$

We define:

$$
\mathbb{S}_{0}:=S\left(\Omega, u^{0}, g\right), \quad \mathbb{S}_{\varepsilon}:=\mathbb{S}\left(\Omega_{\varepsilon}, u^{0} 1_{\Omega_{\varepsilon}}, g 1_{\Omega_{\varepsilon}}\right) 1_{\Omega_{\varepsilon}} \text { and } Q_{\varepsilon}:=(0, T) \times \Omega_{\varepsilon},
$$

for $\Omega, u^{0}, g$ and $\Omega_{\varepsilon}$ as stated in Lemma 2.4. We recall that, since $w \mapsto w 1_{\Omega_{\varepsilon}}$ is an isometric embedding from $\mathcal{W}_{\Omega_{\varepsilon}}$ to $\mathcal{W}_{\Omega}$, we have $\mathbb{S}_{\varepsilon} \in \mathcal{W}_{\Omega}$ and the equality:

$$
\left\|\mathbb{S}_{\varepsilon}\right\|_{\mathcal{W}_{\Omega}}=\left\|\mathbb{S}_{\varepsilon}\right\|_{\mathcal{W}_{\Omega_{\varepsilon}}}
$$

Using the density of $\mathcal{D}\left(Q_{\varepsilon}\right)$ in $\mathcal{W}_{\Omega_{\varepsilon}}$ we find the equality:

$$
\left\|\mathbb{S}_{\varepsilon}(T, \cdot)\right\|_{L^{2}\left(\Omega_{\varepsilon}\right)}^{2}+2 \iint_{Q_{\varepsilon}}\left|\nabla \mathbb{S}_{\varepsilon}\right|^{2}=2 \iint_{Q} g \mathbb{S}_{\varepsilon}+\left\|u^{0}\right\|_{L^{2}\left(\Omega_{\varepsilon}\right)}^{2}
$$

In particular, if we use the Poincaré inequality on $\Omega$ and Cauchy-Schwarz, we obtain for a constant $C(\operatorname{diam}(\Omega))>0$ :

$$
\left\|\mathbb{S}_{\varepsilon}\right\|_{L^{2}\left(0, T ; H_{0}^{1}\left(\Omega_{\varepsilon}\right)\right)} \leq C\left(\|g\|_{L^{2}\left(Q_{\varepsilon}\right)}+\left\|u^{0}\right\|_{L^{2}\left(\Omega_{\varepsilon}\right)}\right) \leq C\left(\|g\|_{L^{2}(Q)}+\left\|u^{0}\right\|_{L^{2}(\Omega)}\right) .
$$

In addition, looking at $2.11_{1}$ we obtain that $\partial_{t} \mathbb{S}_{\varepsilon} \in L^{2}\left(0, T ; H^{-1}\left(\Omega_{\varepsilon}\right)\right)$ and the existence of a constant $C(\operatorname{diam}(\Omega))>0$ such that:

$$
\left\|\mathbb{S}_{\varepsilon}\right\|_{\mathcal{W}_{\Omega_{\varepsilon}}} \leq C\left(\|g\|_{L^{2}(Q)}+\left\|u^{0}\right\|_{L^{2}(Q)}\right)
$$

Consequently, $\mathbb{S}_{\varepsilon}$ is uniformly bounded in $\mathcal{W}_{\Omega}$ due to 2.5 ; thus, $\mathbb{S}_{\varepsilon}$ has at least a weakly convergent sequence in $\mathcal{W}_{\Omega}$. Moreover, thanks to Item 1 of Lemma 2.5, we have that the sequence is convergent in $L^{2}(Q)$.

In order to end the proof, it suffices to see that for all $u \in \mathcal{W}_{\Omega}$ such that there is a sequence $\mathbb{S}_{\varepsilon_{i}}$ satisfying that $\mathbb{S}_{\varepsilon_{i}} \rightarrow u$ in $\mathcal{W}_{\Omega}$ (with $\varepsilon_{i} \rightarrow 0$ ), then $u=\mathbb{S}_{0}$. To get the equality, we prove that $u$ satisfies 2.4 for $\left(\tilde{\Omega}, \tilde{u}^{0}, \tilde{g}\right)=\left(\Omega, u^{0}, g\right)$ :

- In order to prove the variational condition of 2.4 , we pick $\phi \in \mathcal{D}(Q)$. We remark that $d\left(\operatorname{supp}_{x} \phi, \partial \Omega\right)>0$, for:

$$
\operatorname{supp}_{x} \phi:=\overline{\{x: \exists t \in[0, T]: \phi(t, x) \neq 0\}} .
$$


In particular, if $i$ is sufficiently large, we have that $\operatorname{supp}_{x} \phi \subset \subset \Omega_{\varepsilon_{i}}$. Consequently, by $2.4{ }_{1}$ (for $\left.\left(\tilde{\Omega}, \tilde{u}^{0}, \tilde{g}\right)=\left(\Omega_{\varepsilon_{i}}, u^{0} 1_{\Omega_{\varepsilon_{i}}}, g 1_{\Omega_{\varepsilon_{i}}}\right)\right)$ and by taking into account the support of $\phi$, we obtain for $i$ large enough the equality:

$$
\int_{0}^{T}\left\langle\partial_{t} \mathbb{S}_{\varepsilon_{i}}, \phi\right\rangle_{H^{-1}(\Omega), H_{0}^{1}(\Omega)}+\iint_{Q} \nabla \mathbb{S}_{\varepsilon_{i}} \cdot \nabla \phi=\iint_{Q} g \phi .
$$

Thus, if we take the weak limit in $\mathcal{W}_{\Omega}$, we get that:

$$
\int_{0}^{T}\left\langle\partial_{t} u, \phi\right\rangle_{H^{-1}(\Omega), H_{0}^{1}(\Omega)}+\iint_{Q} \nabla u \cdot \nabla \phi=\iint_{Q} g \phi .
$$

Since $\phi$ is arbitrary, $u$ satisfies $(2.4)_{1}$.

- As for the initial condition of (2.4), we recall that because of Item 2 of Lemma 2.5 .

$$
w \in \mathcal{W} \mapsto w(0, \cdot) \in L^{2}(\Omega),
$$

is a continuous operator. Therefore, since the weak limit is preserved by linear continuous operators between Hilbert spaces, we have that $\mathbb{S}_{\varepsilon_{i}}(0, \cdot) \rightarrow u(0, \cdot)$ in $L^{2}(\Omega)$. Moreover, $\mathbb{S}_{\varepsilon_{i}}(0, \cdot)=u^{0} 1_{\Omega_{\varepsilon_{i}}} \rightarrow u^{0}$ in $L^{2}(\Omega)$ by Remark 2.3 . Consequently, from the uniqueness of the weak limit we obtain that $u(0, \cdot)=u^{0}$.

In order to prove a Carleman inequality for $\Omega$, the approximation must be made not only by $C^{2}$ domains, but also these domains must be compatible with the function $\eta$ in the following way:

Definition 2.6. Let $\Omega$ be a domain, let $\omega \subset \Omega$ be a subdomain and let $\eta$ be a real valued function satisfying:

$$
\eta \in C^{2}(\bar{\Omega}), \quad \inf _{\Omega \backslash \omega}|\nabla \eta|>0
$$

We say that $\tilde{\Omega}$ is a compatible open set with respect to $\Omega, \omega$ and $\eta$ if we have that:

$$
\begin{aligned}
& \tilde{\Omega} \text { is a Lipschitz open set such that } \omega \subset \tilde{\Omega} \subset \Omega, \\
& \qquad \begin{array}{c}
\mathcal{D}_{\tilde{\Omega}}(\Delta) \subset H^{2}(\tilde{\Omega}) \text { continuously, } \\
\partial_{\tilde{n}} \eta \leq 0 \text { on } \partial \tilde{\Omega} .
\end{array}
\end{aligned}
$$

Remark 2.7. It is possible for $\eta$ to take negative values. Indeed, in [19] they do not need that $\eta \geq 0$ for proving the controllability of the heat equation with Dirichlet boundary conditions. They need it, though, to prove the controllability of other systems, like the Stokes equation or the heat equation with Neumann boundary conditions. 
Remark 2.8. One consequence of Definition 2.6 is that $\tilde{\Omega}$ is connected. Indeed, if $\tilde{\Omega}_{i}$ is a connected component, then $\omega \cap \tilde{\Omega}_{i} \neq \emptyset$. Otherwise, because of 2.9 and $2.6{ }_{2}$ the maximum of $\eta$ in $\tilde{\Omega}_{i}$ is not reached on the boundary (the tangential derivative of $\eta$ is non-null), but again because of $(2.6)_{2}$ the maximum is not reached in the interior, getting an absurd. Consequently, all the connected components of $\tilde{\Omega}$ intersect $\omega$. Since $\omega$ is connected, $\tilde{\Omega}$ has only one connected component, so $\tilde{\Omega}$ is connected.

Remark 2.9. Every $C^{2}$ domain satisfies 2.8). Moreover, there are some Lipschitz domains which also satisfy it, like $(0,1)^{d}$ or, in general, any cartesian product of $C^{2}$ domains.

Let us now state the Carleman inequality for domains which satisfy $(2.7)-(2.9)$ :

Lemma 2.10. Let $\Omega$ be a Lipschitz domain, $\omega \subset \Omega$ be a subdomain, $\eta$ be a function satisfying (2.6) and $m \geq 1$. Then, there is $C>0$ such that for all $T>0, \tilde{\Omega}$ satisfying (2.7)-(2.9), $g \in L^{2}(\tilde{Q}), u^{T} \in L^{2}(\tilde{\Omega}), k>2(m+1)\|\eta\|_{L^{\infty}(\Omega)}$, and for the weights defined in (1.9), we have that:

$$
\begin{aligned}
s^{3} \lambda^{4} \iint_{\tilde{Q}} e^{-2 s \alpha} \xi^{3}|u|^{2}+s \lambda^{2} \iint_{\tilde{Q}} e^{-2 s \alpha} \xi|\nabla u|^{2}+s^{-1} \iint_{\tilde{Q}} e^{-2 s \alpha} \xi^{-1}\left(|\Delta u|^{2}+\left|u_{t}\right|^{2}\right) \\
\leq C\left(s^{3} \lambda^{4} \iint_{Q_{\omega}} e^{-2 s \alpha} \xi^{3}|u|^{2}+\iint_{\tilde{Q}} e^{-2 s \alpha}|g|^{2}\right)
\end{aligned}
$$

for any $\lambda \geq C, s \geq C\left(T^{m}+T^{2 m}\right)$ and for $u$ the solution of:

$$
\begin{cases}-u_{t}-\Delta u=g & \text { in } \tilde{Q}, \\ u=0 & \text { on } \tilde{\Sigma}, \\ u(T, \cdot)=u^{T} & \text { on } \tilde{\Omega} .\end{cases}
$$

Proof. Lemma 2.10 is a direct consequence of the proof of the usual Carleman estimate, which can be found for instance in [19] and [17]. We remark that since $\inf _{\Omega \backslash \omega}|\nabla \eta|>0$, there exists a domain $\omega_{0} \subset \subset \omega$ which satisfies $\inf _{\Omega \backslash \omega_{0}}|\nabla \eta|>0$. The only additional difficulty is to prove that the constant $C$ is independent of $\tilde{\Omega}$. Indeed, the proofs presented in [19] and [17] show that $C$ depends continuously only on:

- $\|\eta\|_{C^{2}(\bar{\Omega})}$ (which can be bounded by $\left.\|\eta\|_{C^{2}(\bar{\Omega})}\right)$,

- $m($ see 1.10$)$,

- $\inf _{\tilde{\Omega} \backslash \omega_{0}}|\nabla \eta|$ (which can be inferiorly bounded by $\inf _{\Omega \backslash \omega_{0}}|\nabla \eta|>0$ ),

- $\omega$ and $\omega_{0}$. 
Now we are ready to prove a Carleman inequality for domains that can be approximated properly by regular domains:

Lemma 2.11. Let $\Omega$ be a Lipschitz domain, $\omega \subset \Omega$ be a subdomain, $\eta$ be a function satisfying (2.6) and $m \geq 1$. Let us suppose that there are $\varepsilon_{0}>0$ and $\left(\Omega_{\varepsilon}\right)_{\varepsilon \in\left(0, \varepsilon_{0}\right)}$ satisfying (2.7)-(2.9) and such that $\Omega_{\varepsilon} \rightarrow_{d} \Omega$. Then, there is $C>0$ such that for all $T>0, g \in L^{2}(Q), u^{T} \in L^{2}(\Omega)$, $k>2(m+1)\|\eta\|_{L^{\infty}(\Omega)}$, and for the weights defined in (1.9), we have that:

$$
\begin{array}{r}
s^{3} \lambda^{4} \iint_{Q} e^{-2 s \alpha} \xi^{3}|u|^{2}+s \lambda^{2} \iint_{Q} e^{-2 s \alpha} \xi|\nabla u|^{2}+s^{-1} \iint_{Q} e^{-2 s \alpha} \xi^{-1}\left(|\Delta u|^{2}+\left|u_{t}\right|^{2}\right) \\
\leq C\left(s^{3} \lambda^{4} \iint_{Q_{\omega}} e^{-2 s \alpha} \xi^{3}|u|^{2}+\iint_{Q} e^{-2 s \alpha}|g|^{2}\right),
\end{array}
$$

for any $\lambda \geq C, s \geq C\left(T^{m}+T^{2 m}\right)$ and for $u$ the solution of:

$$
\begin{cases}-u_{t}-\Delta u=g & \text { in } Q, \\ u=0 & \text { on } \Sigma, \\ u(T, \cdot)=u^{T} & \text { on } \Omega .\end{cases}
$$

In order to prove Lemma 2.11 we first get the estimate on the zero-order term, and then get the higher order terms as in [15] and [16].

Proof. By Lemma 2.10 we have that for all $m \geq 1$ there is $C>0$ such that for all $T>0$, $u^{T} \in L^{2}(\Omega), g \in L^{2}(Q)$, and $\varepsilon \in\left(0, \varepsilon_{0}\right)$ :

$$
s^{3} \lambda^{4} \iint_{Q} e^{-2 s \alpha} \xi^{3}\left|u^{\varepsilon} 1_{\Omega_{\varepsilon}}\right|^{2} \leq C\left(s^{3} \lambda^{4} \iint_{Q_{\omega}} e^{-2 s \alpha} \xi^{3}\left|u^{\varepsilon}\right|^{2}+\iint_{Q} e^{-2 s \alpha}\left|g 1_{\Omega_{\varepsilon}}\right|^{2}\right),
$$

for any $\lambda \geq C, s \geq C\left(T^{m}+T^{2 m}\right)$ and for:

$$
u^{\varepsilon}(t, x):=\mathbb{S}\left(\Omega_{\varepsilon}, u^{T} 1_{\Omega_{\varepsilon}}, g(T-\cdot, \cdot) 1_{\Omega_{\varepsilon}}\right)(T-t, x) .
$$

Considering Lemma 2.4. we can take the limit in 2.13) and get the estimate:

$$
s^{3} \lambda^{4} \iint_{Q} e^{-2 s \alpha} \xi^{3}|u|^{2} \leq C\left(s^{3} \lambda^{4} \iint_{Q_{\omega}} e^{-2 s \alpha} \xi^{3}|u|^{2}+\iint_{Q} e^{-2 s \alpha}|g|^{2}\right) .
$$

Next, multiplying $2.12{ }_{1}$ by $s \lambda^{2} e^{-2 s \alpha} \xi u$ we get the term of $\nabla u$. Indeed, integrating by parts we get that:

$$
\begin{array}{r}
s \lambda^{2} \iint_{Q} e^{-2 s \alpha} \xi|\nabla u|^{2}=s \lambda^{2} \iint_{Q} e^{-2 s \alpha} g u-s \lambda^{3} \iint_{Q} e^{-2 s \alpha} \xi \nabla \eta \cdot \nabla u u+2 s^{2} \lambda^{3} \iint_{Q} e^{-2 s \alpha} \xi^{2} \nabla \eta \cdot \nabla u u \\
-\frac{s \lambda^{2}}{2} \iint_{Q} e^{-2 s \alpha} \xi_{t}|u|^{2}+s^{2} \lambda^{2} \iint_{Q} e^{-2 s \alpha} \alpha_{t} \xi|u|^{2} . \quad(2.15)
\end{array}
$$


By doing weighted Cauchy-Schwarz estimates in 2.15) and considering (1.10) and 2.14 we get for any $\lambda \geq C$ and $s \geq C\left(T^{m}+T^{2 m}\right)$ that:

$$
s \lambda^{2} \iint_{Q} e^{-2 s \alpha} \xi|\nabla u|^{2} \leq C\left(s^{3} \lambda^{4} \iint_{Q_{\omega}} e^{-2 s \alpha} \xi^{3}|u|^{2}+\iint_{Q} e^{-2 s \alpha}|g|^{2}\right) .
$$

To continue with, we assume that $u^{T} \in H_{0}^{1}(\Omega)$. Multiplying $2.12{ }_{1}$ by $-s^{-1} e^{-2 s \alpha} \xi^{-1} u_{t}$ we obtain that:

$$
\begin{aligned}
& s^{-1} \iint_{Q} e^{-2 s \alpha} \xi^{-1}\left|u_{t}\right|^{2} \\
& =-s^{-1} \iint_{Q} e^{-2 s \alpha} \xi^{-1} g u_{t}-\frac{1}{2} s^{-1} \iint_{Q}\left(e^{-2 s \alpha} \xi^{-1}\right)_{t}|\nabla u|^{2}+s^{-1} \iint_{Q} \nabla\left(e^{-2 s \alpha} \xi^{-1}\right) \cdot \nabla u u_{t} .
\end{aligned}
$$

We have used that the solutions of the heat equation with initial value in $H_{0}^{1}(\Omega)$ and source term in $L^{2}(Q)$ belongs to the closure of $H^{1}\left(0, T ; H_{0}^{1}(\Omega)\right) \cap L^{2}(0, T ; \mathcal{D}(\Delta))$ with norm $H^{1}\left(0, T ; L^{2}(\Omega)\right) \cap$ $L^{2}(0, T ; \mathcal{D}(\Delta))$ when $\Omega$ is Lipschitz. Using weighted Cauchy-Schwarz inequalities, 1.10 and 2.16) we find for any $\lambda \geq C$ and $s \geq C\left(T^{m}+T^{2 m}\right)$ that:

$$
s^{-1} \iint_{Q} e^{-2 s \alpha} \xi^{-1}\left|u_{t}\right|^{2} \leq C\left(s^{3} \lambda^{4} \iint_{Q_{\omega}} e^{-2 s \alpha} \xi^{3}|u|^{2}+\iint_{Q} e^{-2 s \alpha}|g|^{2}\right) .
$$

Finally, considering that $-\Delta u=u_{t}+g$ we obtain from (2.18) that:

$$
s^{-1} \iint_{Q} e^{-2 s \alpha} \xi^{-1}|\Delta u|^{2} \leq C\left(s^{3} \lambda^{4} \iint_{Q_{\omega}} e^{-2 s \alpha} \xi^{3}|u|^{2}+\iint_{Q} e^{-2 s \alpha}|g|^{2}\right) .
$$

So, combining (2.14), 2.16), 2.18 and (2.19) we obtain 2.11) for all $u^{T} \in H_{0}^{1}(\Omega)$. Finally, we obtain 2.11 for all $u^{T} \in L^{2}(\Omega)$ by density.

Finally, we remark that we can extend the result presented in Lemma 2.11 to situations in which there is a source term in $L^{2}\left(0, T ; H^{-1}(\Omega)\right)$ reproducing step by step the proof given in 24] (the reader can consult for instance [1] and [5] to see that the results of the Sobolev spaces used in [24] remain true when $\Omega$ is Lipschitz):

Proposition 2.12. Let $\Omega$ be a Lipschitz domain, $\omega \subset \Omega$ be a subdomain, $\eta$ be a function satisfying (2.6) and $m \geq 1$. Let us suppose that there are $\varepsilon_{0}>0$ and $\left(\Omega_{\varepsilon}\right)_{\varepsilon \in\left(0, \varepsilon_{0}\right)}$ satisfying (2.7)-(2.9) and such that $\Omega_{\varepsilon} \rightarrow_{d} \Omega$. Then, there is $C>0$ such that for all $T>0, g \in L^{2}(Q)$, $G \in\left(L^{2}(Q)\right)^{d}, u^{T} \in L^{2}(\Omega), k>2(m+1)\|\eta\|_{L^{\infty}(\Omega)}$, and for the weights defined in $[1.9)$, we have that:

$$
\begin{aligned}
& s^{3} \lambda^{4} \iint_{Q} e^{-2 s \alpha} \xi^{3}|u|^{2}+s \lambda^{2} \iint_{Q} e^{-2 s \alpha} \xi|\nabla u|^{2} \\
& \leq C\left(s^{3} \lambda^{4} \iint_{Q_{\omega}} e^{-2 s \alpha} \xi^{3}|u|^{2}+\iint_{Q} e^{-2 s \alpha}|g|^{2}+s^{2} \lambda^{2} \iint_{Q} e^{-2 s \alpha} \xi^{2}|G|^{2}\right),
\end{aligned}
$$


for any $\lambda \geq C, s \geq C\left(T^{m}+T^{2 m}\right)$ and for $u$ the solution of:

$$
\begin{cases}-u_{t}-\Delta u=g+\nabla \cdot G & \text { in } Q, \\ u=0 & \text { on } \Sigma, \\ u(T, \cdot)=u^{T} & \text { on } \Omega .\end{cases}
$$

Remark 2.13. As the source term in 2.21 is just in $L^{2}\left(0, T ; H^{-1}(\Omega)\right)$, we do not (and cannot) have neither the Laplacian nor the time derivative on the left-hand side of 2.20).

Remark 2.14. The constant $C$ in Lemma 2.11 and Proposition 2.12 is independent of the sequence $\Omega_{\varepsilon}$.

\section{Proof of Theorem 1.6}

This section is split in three parts: in Subsection 3.1 we prove Theorem 1.6 in the case $\omega \subset \mathfrak{C}$, in Subsection 3.2 we prove Theorem 1.6 in the case $\omega \subset \Omega \backslash \overline{\mathfrak{C}}$, and in Subsection 3.3 we make some comments about some technical motivations and possible extensions.

\subsection{The case $\omega \subset \mathfrak{C}$}

Let $\Omega$ be a canonically oriented pseudo-cylinder (see Definition 1.1 ) and let $\omega \subset \mathfrak{C}$. By taking a smaller control domain if necessary, we can suppose that:

$$
\omega=B(\bar{x}, r) \times(\bar{z}-r, \bar{z}+r) \subset \subset \mathfrak{C}
$$

for some $r>0$ and some $(\bar{x}, \bar{z}) \in \Omega$ such that:

$$
\bar{z}<\inf _{\mathfrak{B}} H
$$

We construct an auxiliary function $\eta$ satisfying $(2.6)$. Considering that $\mathfrak{B}$ is $C^{2}$ and 1.1 , there is $\eta_{\mathfrak{B}} \in C^{2}(\overline{\mathfrak{B}})$ satisfying:

$$
\eta_{\mathfrak{B}}=0 \text { on } \partial \mathfrak{B}, \quad \eta_{\mathfrak{B}}>0 \text { in } \mathfrak{B}, \quad \inf _{\overline{\mathfrak{B}} \backslash B(\bar{x}, r)}\left|\nabla \eta_{\mathfrak{B}}\right|>0 .
$$

One important consequence of $(3.3)$ is the inequality:

$$
\sup _{\partial \mathfrak{B}} \partial_{n_{\mathfrak{B}}} \eta_{\mathfrak{B}}<0
$$

Indeed, using 3.3$)_{1}$ we have that the tangential derivative of $\eta_{\mathfrak{B}}$ is null on $\partial \mathfrak{B}$. Thus, using $3.3)_{2}$ and that $B(\bar{x}, r) \subset \subset \mathfrak{B}$, we obtain that $\partial_{n_{\mathfrak{B}}} \eta_{\mathfrak{B}}(x) \neq 0$ for all $x \in \partial \mathfrak{B}$. Moreover, from $3.3)_{1}$ and 3.3$)_{2}$ we find that $\partial_{n_{\mathfrak{B}}} \eta_{\mathfrak{B}}(x)<0$ for all $x \in \partial \mathfrak{B}$, which implies 3.4 by compactness. 


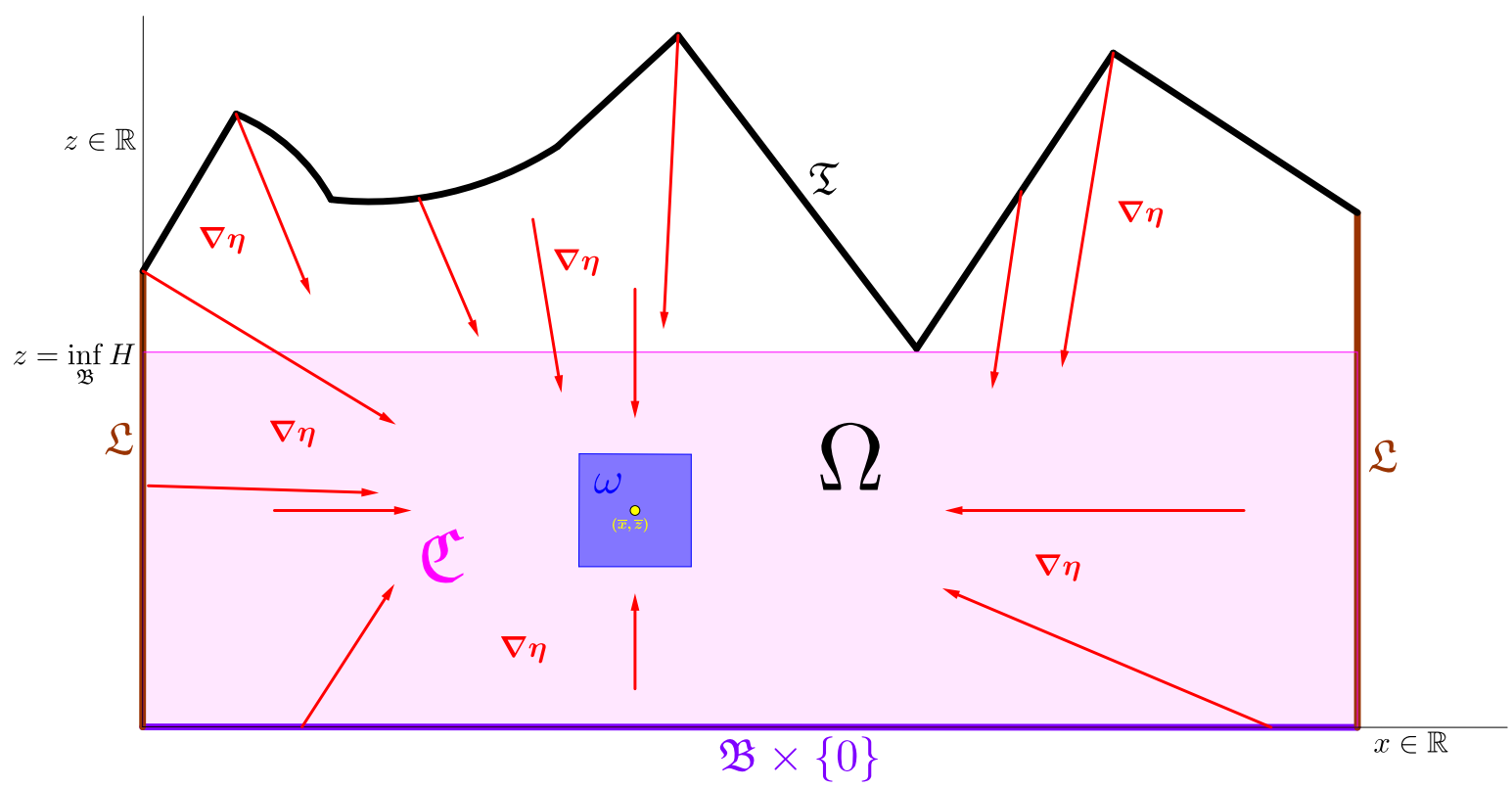

Figure 2: A canonically oriented pseudo-cylinder with its auxiliary function.

So, we define in $\bar{\Omega}$ :

$$
\eta(x, z):=\eta_{\mathfrak{B}}(x)-\mathfrak{c}(z-\bar{z})^{2}
$$

for $\mathfrak{c}$ a large positive constant to be fixed later on. It is evident that $\eta \in C^{2}(\bar{\Omega})$. As $\nabla \eta=$ $\left(\nabla \eta_{\mathfrak{B}},-2 \mathfrak{c}(z-\bar{z})\right)$, it follows from $(3.1)$ and 3.3$)_{3}$ the inequality:

$$
\inf _{\Omega \backslash \omega}|\nabla \eta|>0
$$

Example 3.1. See Figure 2 for an illustration of a control domain and of $\nabla \eta$ in the pseudocylinder introduced in Example 1.3.

With the above auxiliary function we obtain the following Carleman inequality:

Proposition 3.2. Let $\Omega$ be a canonically oriented pseudo-cylinder, $\omega$ be given by (3.1) and $m \geq 1$. Then, there exists $\mathfrak{c}_{0}\left(\Omega, \omega, \eta_{\mathfrak{B}}\right)>0$ such that if $\eta$ is defined as in (3.5) with $\mathfrak{c} \geq \mathfrak{c}_{0}$, there is $C>0$ such that for all $T>0, A \in\left(L^{\infty}(Q)\right)^{d+1}, a \in L^{\infty}(Q), g \in L^{2}(Q), G \in\left(L^{2}(Q)\right)^{d+1}$, $u^{T} \in L^{2}(\Omega), k>2(m+1)\|\eta\|_{L^{\infty}(\Omega)}$, and for the weights defined in (1.9), we have that:

$$
\begin{aligned}
& s^{3} \lambda^{4} \iint_{Q} e^{-2 s \alpha} \xi^{3}|u|^{2}+s \lambda^{2} \iint_{Q} e^{-2 s \alpha} \xi|\nabla u|^{2} \\
& \leq C\left(s^{3} \lambda^{4} \iint_{Q_{\omega}} e^{-2 s \alpha} \xi^{3}|u|^{2}+\iint_{Q} e^{-2 s \alpha}|g|^{2}+s^{2} \lambda^{2} \iint_{Q} e^{-2 s \alpha} \xi^{2}|G|^{2}\right),
\end{aligned}
$$


for any $\lambda \geq C$,

$$
s \geq C\left(T^{m}+T^{2 m}\left(1+\|a\|_{L^{\infty}(Q)}^{2 / 3}+\|A\|_{\left(L^{\infty}(Q)\right)^{d+1}}^{2}\right)\right),
$$

and for $u$ the solution of:

$$
\begin{cases}-u_{t}-\Delta u-\nabla \cdot(A u)+a u=g+\nabla \cdot G & \text { in } Q, \\ u=0 & \text { on } \Sigma, \\ u(T, \cdot)=u^{T} & \text { on } \Omega .\end{cases}
$$

In order to prove Proposition 3.2 we state the following technical result whose proof is postponed to Appendix $\mathrm{A}$.

Proposition 3.3. Let $\Omega$ be a canonically oriented pseudo-cylinder, $\omega$ be given by (3.1) and $\eta_{\mathfrak{B}} \in C^{2}(\overline{\mathfrak{B}})$ satisfying (3.3). Then, there is $\mathfrak{c}_{0}\left(\Omega, \omega, \eta_{\mathfrak{B}}\right)>0$ such that if $\eta$ is given by (3.5) with $\mathfrak{c} \geq \mathfrak{c}_{0}$, there exist $\varepsilon_{0}\left(\Omega, \omega, \eta_{\mathfrak{B}}, \mathfrak{c}\right)>0$ and some domains $\left(\Omega_{\varepsilon}\right)_{\varepsilon \in\left(0, \varepsilon_{0}\right)}$ satisfying (2.7)-2.9) and:

$$
\Omega_{\varepsilon} \rightarrow_{d} \Omega .
$$

Proof of Proposition 3.2. Using Propositions 3.3 and 2.12, we obtain that for all $\lambda \geq C$ and $s \geq C\left(T^{m}+T^{2 m}\right):$

$$
\begin{aligned}
& s^{3} \lambda^{4} \iint_{Q} e^{-2 s \alpha} \xi^{3}|u|^{2}+s \lambda^{2} \iint_{Q} e^{-2 s \alpha} \xi|\nabla u|^{2} \\
& \leq C\left(s^{3} \lambda^{4} \iint_{Q_{\omega}} e^{-2 s \alpha} \xi^{3}|u|^{2}+\iint_{Q} e^{-2 s \alpha}\left(|g|^{2}+|a u|^{2}\right)+s^{2} \lambda^{2} \iint_{Q} e^{-2 s \alpha} \xi^{2}\left(|G|^{2}+|A u|^{2}\right)\right) \\
& \leq C\left(s^{3} \lambda^{4} \iint_{Q_{\omega}} e^{-2 s \alpha} \xi^{3}|u|^{2}+\iint_{Q} e^{-2 s \alpha}|g|^{2}+s^{2} \lambda^{2} \iint_{Q} e^{-2 s \alpha} \xi^{2}|G|^{2}\right. \\
& \left.\quad+\|a\|_{L^{\infty}(Q)}^{2} T^{6 m} \iint_{Q} e^{-2 s \alpha} \xi^{3}|u|^{2}+s^{2} \lambda^{2}\|A\|_{L^{\infty}(Q)}^{2} T^{2 m} \iint_{Q} e^{-2 s \alpha} \xi^{3}|u|^{2}\right) .
\end{aligned}
$$

Thus, taking $\lambda \geq C$ and $s$ satisfying (3.7) we can absorb the the last two terms in the right-hand side of $(3.10)$.

Remark 3.4. It is a classical result (see, for instance, [35], [29], [19] and [18]) that Proposition 3.2 (taking $m=1$ ) implies that Theorem 1.6 is true for all canonically oriented pseudo-cylinders and all $\omega$ given by (3.1). By Remark 1.7 this implies Theorem 1.6 for all pseudo-cylinders and all $w \subset \mathfrak{C}$.

\subsection{The case $\omega \subset \Omega \backslash \overline{\mathfrak{C}}$}

Let $\Omega$ be a canonically oriented pseudo-cylinder and let $\omega \subset \Omega \backslash \overline{\mathfrak{C}}$. For this case we suppose, by making again the control domain smaller if necessary, that:

$$
\omega=B(\tilde{x}, r) \times(\tilde{z}-r, \tilde{z}+r) \subset \subset \Omega \backslash \overline{\mathfrak{C}},
$$






Figure 3: A canonically oriented pseudo-cylinder with a control domain in $\Omega \backslash \overline{\mathfrak{C}}$.

for some $(\tilde{x}, \tilde{z}) \in \Omega \backslash \overline{\mathfrak{C}}$ such that:

$$
\tilde{z}>\inf _{\mathfrak{B}} H
$$

and some:

$$
r \in\left(0, \inf _{\mathfrak{B}} \frac{H}{4}\right)
$$

such that:

$$
\tilde{\Omega}:=B(\tilde{x}, 3 r) \times(0, \tilde{z}+3 r) \subset \Omega .
$$

In this case we cannot consider an auxiliary function similar to (3.5) because now we have that $\mathfrak{T} \cap\{(x, z): z=\tilde{z}\} \neq \emptyset$. However, thanks to Proposition 3.2 our problem can be seen as a problem of transmitting the estimate from one control domain to another. Indeed, we define:

$$
\omega^{*}:=B(\bar{x}, r) \times(\bar{z}-r, \bar{z}+r),
$$

for $\bar{x}:=\tilde{x}$ and $\bar{z}:=3 r$. Thus, considering also 3.12 we have that:

$$
\omega^{*}=B(\tilde{x}, r) \times(2 r, 4 r) \subset \subset \tilde{\Omega} \cap \mathfrak{C}
$$

Example 3.5. See Figure 3 for an illustration of the pseudo-cylinder given in Example 1.3 when $\omega \subset \subset \Omega \backslash \overline{\mathfrak{C}}$. 
We fix $m \geq 1$ and define $\eta$ as in (3.5) with $\mathfrak{c} \geq \mathfrak{c}_{0}\left(\Omega, \omega^{*}, \eta_{\mathfrak{B}}\right)$. We obtain by Proposition 3.2 a constant $C>0$ such that for all $T>0, A \in\left(L^{\infty}(Q)\right)^{d+1}, a \in L^{\infty}(Q), g \in L^{2}(Q)$, $G \in\left(L^{2}(Q)\right)^{d+1}, u^{T} \in L^{2}(\Omega)$, and $k>2(m+1)\|\eta\|_{L^{\infty}(\Omega)}$ we have that:

$$
\begin{aligned}
& s^{3} \lambda^{4} \iint_{Q} e^{-2 s \alpha} \xi^{3}|u|^{2}+s \lambda^{2} \iint_{Q} e^{-2 s \alpha} \xi|\nabla u|^{2} \\
& \quad \leq C\left(s^{3} \lambda^{4} \iint_{Q_{\omega^{*}}} e^{-2 s \alpha} \xi^{3}|u|^{2}+\iint_{Q} e^{-2 s \alpha}|g|^{2}+s^{2} \lambda^{2} \iint_{Q} e^{-2 s \alpha} \xi^{2}|G|^{2}\right),
\end{aligned}
$$

for $\lambda \geq C, s$ satisfying (3.7) and $u$ the solution of (3.8).

Next, we estimate the weighted $L^{2}\left(Q_{\omega^{*}}\right)$-norm by a weighted $L^{2}\left(Q_{\omega}\right)$-norm. To do this, we consider a function whose maximum is in $\omega$ but which is large in $\omega^{*}$. Specifically, we consider:

$$
\tilde{\eta}(x, z):=-\tilde{\eta}_{1}(|x-\tilde{x}|)-\tilde{\eta}_{2}(z)+2 \sup _{\Omega}|\eta|
$$

for $\tilde{\eta}_{1}$ a positive $C^{\infty}$ function supported in $(r / 2,+\infty)$ such that:

$$
\left\{\begin{array}{l}
\inf _{(r,+\infty)} \tilde{\eta}_{1}^{\prime}>0 \\
\tilde{\eta}_{1}<\sup _{\Omega}|\eta| / 2 \text { in }(r / 2, r) \\
\tilde{\eta}_{1}>3 \sup _{\Omega}|\eta| \text { in }(2 r, 3 r)
\end{array}\right.
$$

and for $\tilde{\eta}_{2}$ a positive $C^{\infty}$ function such that:

$$
\left\{\begin{array}{l}
\sup _{(0, \tilde{z}-r)} \tilde{\eta}_{2}^{\prime}<0 \\
\inf _{(\tilde{z}+r, \tilde{z}+3 r)} \tilde{\eta}_{2}^{\prime}>0 \\
\tilde{\eta}_{2}<\sup _{\Omega}|\eta| / 2 \text { in }(2 r, 4 r) \\
\tilde{\eta}_{2}>3 \sup _{\Omega}|\eta| \text { in }(0, r) \cup(\tilde{z}+2 r, \tilde{z}+3 r) .
\end{array}\right.
$$

We remark that $\tilde{\eta} \in C^{2}(\overline{\tilde{\Omega}})$ and satisfies:

$$
\partial_{n} \tilde{\eta}<0 \text { on } \partial \tilde{\Omega}, \quad \inf _{\tilde{\Omega} \backslash \omega}|\nabla \tilde{\eta}|>0 .
$$

Moreover, from (3.14), 3.17) and (3.18) we get that:

$$
\left\{\begin{array}{l}
\eta \leq \tilde{\eta} \text { in } \omega^{*} \\
\tilde{\eta} \leq \eta \text { in } \tilde{\Omega} \backslash(B(\tilde{x}, 2 r) \times(r, \tilde{z}+2 r)) .
\end{array}\right.
$$


With the function $\tilde{\eta}$ we can define the weights $\tilde{\alpha}$ and $\tilde{\xi}$ as in 1.9 . Let us fix:

$$
k>2(m+1) \max \left\{\|\tilde{\eta}\|_{L^{\infty}(\Omega)},\|\eta\|_{L^{\infty}(\Omega)}\right\}=2(m+1)\|\tilde{\eta}\|_{L^{\infty}(\Omega)} .
$$

Thanks to 3.20 and Remark 1.12 we have that:

$$
\begin{cases}\xi \leq \tilde{\xi} ; \quad-\alpha \leq-\tilde{\alpha} \quad \text { in } Q_{\omega^{*}}, \\ \tilde{\xi} \leq \xi ; \quad-\tilde{\alpha} \leq-\alpha \quad \text { in }(0, T) \times(\tilde{\Omega} \backslash(B(\tilde{x}, 2 r) \times(r, \tilde{z}+2 r))) .\end{cases}
$$

Next, we consider that $u$ is the solution of $(3.8)$ and that $\chi(x, z)$ is a positive regular cut-off function such that:

$$
\operatorname{supp}(\chi) \subset \subset \tilde{\Omega}, \quad \chi=1 \text { in } B(\tilde{x}, 2 r) \times(r, \tilde{z}+2 r)
$$

Then, $v:=\chi u$ is the solution of:

$$
\begin{cases}-v_{t}-\Delta v-\nabla \cdot(A v)+a v=F(\nabla \chi, u)+\chi g+\nabla \cdot(\chi G) & \text { in } \tilde{Q} \\ v=0 & \text { on } \tilde{\Sigma} \\ v(T, \cdot)=\chi u^{T} & \text { on } \tilde{\Omega}\end{cases}
$$

for:

$$
F(\nabla \chi, u):=-2 \nabla \chi \cdot \nabla u-\Delta \chi u-(\nabla \chi \cdot A) u-\nabla \chi \cdot G .
$$

Using Proposition $2.12(\tilde{\Omega}$ satisfies 2.8$)$ by Remark 2.9 and $(2.9)$ by (3.19), so it suffices to take $\left.\Omega_{\varepsilon}=\tilde{\Omega}\right)$, we have that there is $C(\tilde{\eta}, m)>0$ such that if $A \in\left(L^{\infty}(Q)\right)^{d+1}, a \in L^{\infty}(Q)$, $g \in L^{2}(Q), G \in\left(L^{2}(Q)\right)^{d+1}, u^{T} \in L^{2}(\Omega), \lambda \geq C$ and $s \geq C\left(T^{m}+T^{2 m}\right):$

$$
\begin{aligned}
s^{3} \lambda^{4} \iint_{\tilde{Q}} e^{-2 s \tilde{\alpha}}(\tilde{\xi})^{3}|v|^{2}+s \lambda^{2} \iint_{\tilde{Q}} e^{-2 s \tilde{\alpha} \tilde{\xi}|\nabla v|^{2}} \leq C\left(s^{3} \lambda^{4} \iint_{Q_{\omega}} e^{-2 s \tilde{\alpha}}(\tilde{\xi})^{3}|\chi u|^{2}\right. \\
\left.\quad+\iint_{\tilde{Q}} e^{-2 s \tilde{\alpha}}|F(\nabla \chi, u)+\chi g-a v|^{2}+s^{2} \lambda^{2} \iint_{\tilde{Q}} e^{-2 s \tilde{\alpha}}(\tilde{\xi})^{2}\left(|\chi G-A v|^{2}\right)\right) .
\end{aligned}
$$

After some easy absorptions and bounds we obtain, from (3.26) for $\lambda \geq C$ and $s$ satisfying (3.7), that:

$$
\begin{aligned}
& s^{3} \lambda^{4} \iint_{\tilde{Q}} e^{-2 s \tilde{\alpha}}(\tilde{\xi})^{3}|\chi u|^{2}+s \lambda^{2} \iint_{\tilde{Q}} e^{-2 s \tilde{\alpha} \tilde{\xi}|\nabla(\chi u)|^{2}} \\
\leq & C\left(s^{3} \lambda^{4} \iint_{Q_{\omega}} e^{-2 s \tilde{\alpha}}(\tilde{\xi})^{3}|\chi u|^{2}+\iint_{\tilde{Q}} e^{-2 s \tilde{\alpha}}\left(|\chi g|^{2}+|F(\nabla \chi, u)|^{2}\right)+s^{2} \lambda^{2} \iint_{\tilde{Q}} e^{-2 s \tilde{\alpha}}(\tilde{\xi})^{2}|\chi G|^{2}\right) .
\end{aligned}
$$


To continue with, as $\chi=1$ in $\omega$ and $\omega^{*}, \chi \in \mathcal{D}(\tilde{\Omega})$, and we have 3.221 , we may combine (3.15) and (3.27) and get that:

$$
\begin{gathered}
s^{3} \lambda^{4} \iint_{Q} e^{-2 s \alpha} \xi^{3}|u|^{2}+s \lambda^{2} \iint_{Q} e^{-2 s \alpha} \xi|\nabla u|^{2}+s^{3} \lambda^{4} \iint_{\tilde{Q}} e^{-2 s \tilde{\alpha}}(\tilde{\xi})^{3}|\chi u|^{2}+s \lambda^{2} \iint_{\tilde{Q}} e^{-2 s \tilde{\alpha} \tilde{\xi}|\nabla(\chi u)|^{2}} \\
\leq C\left(s^{3} \lambda^{4} \iint_{Q_{\omega}} e^{-2 s \tilde{\alpha}}(\tilde{\xi})^{3}|u|^{2}+\iint_{Q} e^{-2 s \alpha}|g|^{2}+\iint_{\tilde{Q}} e^{-2 s \tilde{\alpha}}\left(|\chi g|^{2}+|F(\nabla \chi, u)|^{2}\right)\right. \\
\left.\quad+s^{2} \lambda^{2} \iint_{Q} e^{-2 s \alpha} \xi^{2}|G|^{2}+s^{2} \lambda^{2} \iint_{\tilde{Q}} e^{-2 s \tilde{\alpha}}(\tilde{\xi})^{2}|\chi G|^{2}\right)
\end{gathered}
$$

Next, we recall that $\chi \in C^{2}(\bar{\Omega})$, that:

$$
\operatorname{supp}(\Delta \chi), \operatorname{supp}(\nabla \chi) \subset \tilde{\Omega} \backslash(B(\tilde{x}, 2 r) \times(r, \tilde{z}+2 r)),
$$

and $(3.22)_{2}$. These assertions imply that by taking $\lambda \geq C$ and $s$ satisfying (3.7) the term of $F(\nabla \chi, u)$ in the right-hand side of $(3.28)$ can be absorbed. Thus, we get:

$$
\begin{gathered}
s^{3} \lambda^{4} \iint_{Q} e^{-2 s \alpha} \xi^{3}|u|^{2}+s \lambda^{2} \iint_{Q} e^{-2 s \alpha} \xi|\nabla u|^{2}+s^{3} \lambda^{4} \iint_{\tilde{Q}} e^{-2 s \tilde{\alpha}}(\tilde{\xi})^{3}|\chi u|^{2}+s \lambda^{2} \iint_{\tilde{Q}} e^{-2 s \tilde{\alpha} \tilde{\xi}|\nabla(\chi u)|^{2}} \\
\leq C\left(s^{3} \lambda^{4} \iint_{Q_{\omega}} e^{-2 s \tilde{\alpha}}(\tilde{\xi})^{3}|u|^{2}+\iint_{Q} e^{-2 s \alpha}|g|^{2}+\iint_{\tilde{Q}} e^{-2 s \tilde{\alpha}}|\chi g|^{2}\right. \\
\left.\quad+s^{2} \lambda^{2} \iint_{Q} e^{-2 s \alpha} \xi^{2}|G|^{2}+s^{2} \lambda^{2} \iint_{\tilde{Q}} e^{-2 s \tilde{\alpha}}(\tilde{\xi})^{2}|\chi G|^{2}\right)
\end{gathered}
$$

Finally, let us state what we have proved:

Proposition 3.6. Let $\Omega$ be a canonically oriented pseudo-cylinder, $\omega$ be given by (3.11), $\Omega$ be given by (3.13), $\eta$ be given by (3.5) (with $\bar{x}:=\tilde{x}$ and $\bar{z}:=3 r$ ), $\tilde{\eta}$ be given by (3.16), $\chi$ be given by 3.23 and $m \geq 1$. Then, there exists a constant $C>0$ such that if $T>0, A \in\left(L^{\infty}(Q)\right)^{d+1}$, $a \in L^{\infty}(Q), g \in L^{2}(Q), G \in\left(L^{2}(Q)\right)^{d+1}, u^{T} \in L^{2}(\Omega), k$ satisfies (3.21), $\lambda \geq C$ and $s$ satisfies (3.7), we have (3.29) for the weights defined in (1.9) and $u$ the solution of (3.8).

Remark 3.7. It is a classical result (see, for instance, [35, [29], [19] and [18]) that Proposition 3.6 (taking $m=1$ ) implies that Theorem 1.6 is true for all canonically oriented pseudo-cylinders and all $\omega$ given by (3.11). Together with Remarks 3.4 and 1.7 , this result ends the proof of Theorem 1.6 (up to proving Proposition 3.3).

\subsection{Further comments}

1. Boundary controllability. If $\Gamma \subset \mathfrak{T}$ (see Remark 1.2 is a relatively open connected subset we can easily prove the null controllability of the heat equation with a control supported in $\Gamma$. Indeed, it suffices to extend the domain over $\Gamma$ as in Figure 4 to some 


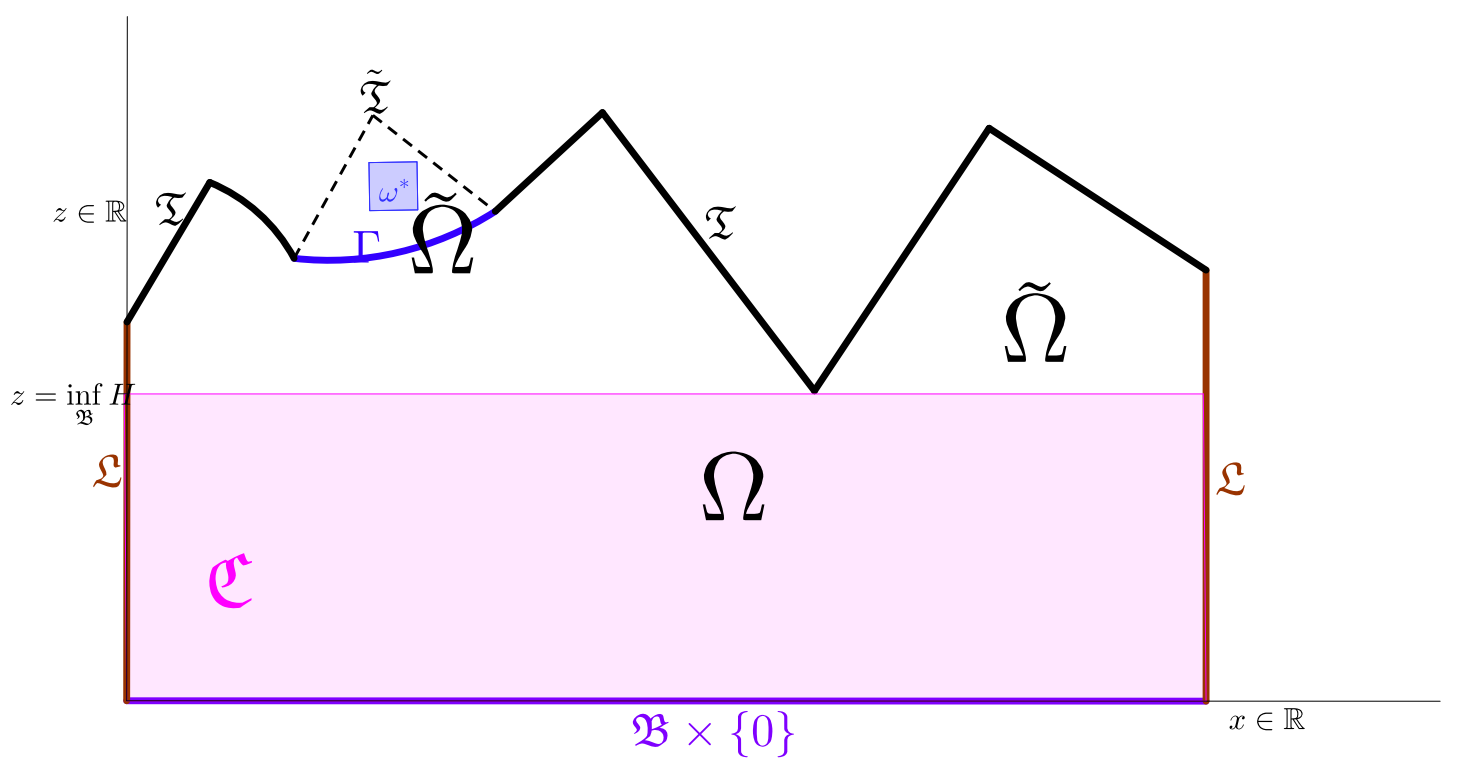

Figure 4: A canonically oriented pseudo-cylinder with a control domain $\Gamma \subset \mathfrak{T}$.

other pseudo-cylinder $\tilde{\Omega}$, use the null controllability by means of an internal control of the heat equation with Dirichlet boundary condition, consider the restriction of the value in $\Gamma$ and use the uniqueness of solutions by transposition of the heat equation. If $\Gamma \subset \mathfrak{L}$ or if $\Gamma \subset \mathfrak{B} \times\{0\}$ this method fails because the extended domain is not a pseudo-cylinder. Thus, for those situations the boundary controllability problem remains open. Moreover, it would also be interesting to obtain Carleman inequalities of the Fursikov-Imanuvilov's type for a control domain $\Gamma \subset \partial \Omega$, which has a local boundary term of $\partial_{n} u$ in the right-hand side of the Carleman estimate.

2. The Carleman estimate when $A$ is more regular and $G=0$. If we have that $\nabla \cdot A \in L^{\infty}(Q)$ and $G=0$ we can add a term of a Laplacian and of a time derivative in the Carleman estimates obtained in Propositions 3.2 and 3.6 by using Lemma 2.11 instead of Proposition 2.12 .

3. Validity of the partial results in any pseudo-cylinder. Even if we have stated and proved the partial results (see Propositions 3.2, 3.3 and 3.6 in canonically oriented pseudo-cylinders, these results hold in any pseudo-cylinder since the Laplacian operator is invariant under rotations (and translations).

4. The dependence of $\mathfrak{c}$ with respect to $\eta_{\mathfrak{B}}$. It seems impossible to approximate a domain $\Omega$ by some subdomains $\Omega_{\varepsilon}$ satisfying (2.7)-(2.9) without having $\partial_{n} \eta \leq 0$ on $\partial \Omega$ (though this is an interesting open question). This implies that if $\Omega$ is a pseudo-cylinder, 
if $\omega$ is given by (3.1) and if $\eta$ is the auxiliary function defined in (3.5), we need to take $\mathfrak{c}$ large enough with respect to $\eta_{\mathfrak{B}}$. Indeed, let us compute $\partial_{n} \eta$ and verify that it is negative on all the subsets of the boundary given by Remark 1.2 .

- On $\mathfrak{B} \times\{0\}$ we have that $\partial_{n} \eta=-\partial_{z} \eta=-2 \mathfrak{c} \bar{z}<0$.

- On $\mathfrak{L}$ we have by (3.4) that $\partial_{n} \eta=\partial_{n_{\mathfrak{B}}} \eta_{\mathfrak{B}}<0$.

- On $\mathfrak{T}$ we find that:

$$
n(x, H(x))=\frac{1}{\sqrt{1+|\nabla H(x)|^{2}}}(-\nabla H(x), 1) .
$$

Thus, on $\mathfrak{T}$ the normal derivative is given by:

$$
\partial_{n} \eta(x, H(x))=\frac{-\nabla H(x) \cdot \nabla \eta_{\mathfrak{B}}(x)-2 \mathfrak{c}(H(x)-\bar{z})}{\sqrt{1+|\nabla H(x)|^{2}}} .
$$

This implies that the condition:

$$
\mathfrak{c} \geq \frac{\|\nabla H\|_{L^{\infty}(\mathfrak{B})}\left\|\nabla \eta_{\mathfrak{B}}\right\|_{C(\overline{\mathfrak{B}})}}{2\left(\inf _{\mathfrak{B}} H(x)-\bar{z}\right)}
$$

is necessary to ensure that $\partial_{n} \eta \leq 0$ on $\mathfrak{T}$ (the denominator in 3.30 is not null by $(3.2)$.

5. Problems when extending this method to Lipschitz domains. A problem that remains open is the null controllability of the heat equation in any Lipschitz domain. A natural attempt following the Fursikov-Imanuvilov approach is to split the domain into a finite amount of pseudo-cylinders and a single compactly included $C^{2}$ subdomain, and then to apply a Carleman estimate in each subdomain (with the help of some cut-off functions). To that end, we need to absorb what is in a neighbourhood $\mathfrak{L}$ (see Remark 1.2 with estimates in some other pseudo-cylinders and then use the same technique as Section 3.2 to pass from a finite number of control domains that are compactly contained in $\Omega$ to a single control domain. This idea could work if we had a function $\eta$ satisfying (2.6) which is much bigger in the interior of the cylinders than close to $\mathfrak{L}$. However, it seems incompatible with picking in 3.5 c large with respect to $\eta_{\mathfrak{B}}$.

6. Semi-linear heat equation. A problem that remains open is the controllability of the semi-linear heat equation stated in pseudo-cylinders with a non-linearity acting also in the first order term. Indeed, we cannot just follow the steps in [8] because they require for $\Omega$ to be $C^{2}$ when they prove that the Carleman estimate implies the null-controllability of the semi-linear heat equation (more specifically, they use that $\mathcal{D}(\Delta) \subset H^{2}(\Omega)$ continuously and the analogous results for $L^{p}$-spaces). 


\section{A Proof of Proposition 3.3}

We recall that $\Omega$ is a canonically oriented pseudo-cylinder, $\omega$ is given by (3.1) and $\eta$ is given by (3.5). Similarly, in this proof we use $c$ and $C$ to denote respectively small and large strictly positive constants that may be different each time and only depend on $\Omega, \omega$ and $\eta_{\mathfrak{B}}$, and $\varepsilon$ is taken as a small strictly positive parameter.

The structure of the proof is the following: first we present an auxiliary result from differential geometry, second we introduce the regularized measure, third we approximate $H$ (see Definition 1.1) by smaller regular functions, fourth we introduce some domains $\Omega_{\varepsilon}$ and prove that they satisfy (3.9), fifth we prove that the domains $\Omega_{\varepsilon}$ satisfy $(2.7)$ and $(2.8)$ for all $\varepsilon<<<\varepsilon_{0}(\Omega, \omega)$, and finally we assume that $\mathfrak{c}>>>\mathfrak{c}_{0}\left(\Omega, \omega, \eta_{\mathfrak{B}}\right)$ and $\varepsilon<<<\varepsilon_{0}\left(\Omega, \omega, \eta_{\mathfrak{B}}, \mathfrak{c}\right)$ and prove $(2.9)$. We recall that once the open sets $\Omega_{\varepsilon}$ satisfy $(2.7)-(2.9)$, Remark 2.8 implies that they are connected.

In the proof we state that several things happen if $\mathfrak{c}>>>\mathfrak{c}_{0}\left(\Omega, \omega, \eta_{\mathfrak{B}}\right)$ and $\varepsilon<<<\varepsilon_{0}\left(\Omega, \omega, \eta_{\mathfrak{B}}, \mathfrak{c}\right)$. We do not calculate explicitly the value of $\mathfrak{c}_{0}\left(\Omega, \omega, \eta_{\mathfrak{B}}\right)$ and of $\varepsilon_{0}\left(\Omega, \omega, \eta_{\mathfrak{B}}, \mathfrak{c}\right)$ the same way we do not calculate explicitly the values of $C$ or $c$. Indeed, note that the number of times that we require such a bound is finite (as $\mathfrak{c}_{0}$ and $\varepsilon_{0}$ do not depend on the specific $(x, z)$ but at most on a finite division of subcases), so it suffices to consider the maximum of all the $\mathfrak{c}_{0}$ and the minimum of all the $\varepsilon_{0}$.

Step 1: an auxiliary result. In order to construct these domains, we need the following basic result from differential geometry, whose proof can be found for instance in [7]:

Lemma A.1. Let $\tilde{\Omega}$ be a domain and let $\Phi: \tilde{\Omega} \mapsto \mathbb{R}$ be a $C^{2}$ function such that $\Phi \leq 0$ on $\partial \tilde{\Omega}$. Then, for all $\theta>0$ satisfying $\Phi^{-1}(\theta,+\infty) \neq \emptyset$ and:

$$
\Phi^{-1}(\{\theta\}) \cap(\nabla \Phi)^{-1}(\{0\})=\emptyset,
$$

we have that $\Phi^{-1}(\theta,+\infty)$ is a $C^{2}$ open set. Moreover, the boundary of $\Phi^{-1}(\theta,+\infty)$ is given by $\Phi^{-1}(\{\theta\})$. Finally, the outward normal unit vector, which we denote by $n_{\theta}$, satisfies on $\Phi^{-1}(\{\theta\})$ :

$$
n_{\theta}(x)=-\frac{\nabla \Phi(x)}{|\nabla \Phi(x)|}
$$

A first approach can be to use Lemma A.1 on the distance function from $(x, z)$ to $\partial \Omega$. Nonetheless, this is not possible because that function is not differentiable. On the one hand, even in regular domains $d(\cdot, \partial \Omega)$ may not be $C^{1}$ in the interior (for instance, in the disk the distance to 
the boundary is not differentiable at its center). On the other hand, near $\mathfrak{T}$ the function $d(\cdot, \partial \Omega)$ is not $C^{1}$ when $\mathfrak{T}$ is just Lipschitz. However, it is possible to construct a function which behaves similarly to the distance. In particular, to face the first problem we regularize the distance with the tools introduced in [28] and to face the second one we approximate $H$ by regular functions.

Step 2: the regularized measure. We define the function:

$$
\mathfrak{d}: x \in \overline{\mathfrak{B}} \mapsto d(x, \partial \mathfrak{B}),
$$

and the sets:

$$
\mathfrak{B}_{\varepsilon}:=\mathfrak{d}^{-1}(\varepsilon,+\infty) .
$$

Since $\mathfrak{B}$ is $C^{2}$, we have for some $\tilde{\varepsilon}(\Omega)>0$ the following properties:

- $\mathfrak{d}$ is a $W^{2, \infty}\left(\mathfrak{d}^{-1}([0, \tilde{\varepsilon}])\right)$ function such that $|\nabla \mathfrak{d}|=1$ in $\mathfrak{d}^{-1}([0, \tilde{\varepsilon}])$.

- For all $x \in \mathfrak{d}^{-1}([0, \tilde{\varepsilon}])$ there is a unique point $P(x)$ such that:

$$
d(x, P(x))=\mathfrak{d}(x) .
$$

Moreover, the function $x \mapsto P(x)$ is continuous in $\mathfrak{d}^{-1}([0, \tilde{\varepsilon}])$.

The proof of these two assertions can be found for instance in [5, Section III.3]. Also, it can be proved easily that for all $\varepsilon<<<\varepsilon_{0}(\Omega)$ the set $\mathfrak{B}_{\varepsilon}$ is a domain satisfying:

$$
\partial \mathfrak{B}_{\varepsilon}=\mathfrak{d}^{-1}(\{\varepsilon\}) .
$$

In order to regularize $\mathfrak{d}$ in the interior we use the function introduced in [28], whose properties can be consulted for instance in [5, Section III.3.3]. Indeed, we consider:

$$
\rho: x \in \overline{\mathfrak{B}} \mapsto \mathbb{R},
$$

which is defined as the only fixed point of $\tau \mapsto G(x, \tau)$, for:

$$
G(x, \tau):=\int_{B(0,1)} \mathfrak{d}\left(x+\frac{\tau}{2} \tilde{x}\right) \varsigma(\tilde{x}) d \tilde{x}
$$

for $\varsigma$ a mollifier in $\mathbb{R}^{d}$; that is, a positive function belonging to $\mathcal{D}(B(0,1))$ such that:

$$
\int_{B(0,1)} \varsigma(x) d x=1
$$


We have that $\rho$ satisfies the following properties, which are proved for instance in [5, Section III.3.3]:

$$
\begin{gathered}
\rho \in W^{2, \infty}(\mathfrak{B}) \cap C^{\infty}(\mathfrak{B}), \\
\rho \geq 0 \text { in } \overline{\mathfrak{B}} \text { and } \rho=0 \text { if and only if } x \in \partial \mathfrak{B}, \\
\frac{1}{2} \leq \frac{\mathfrak{d}(x)}{\rho(x)} \leq \frac{3}{2}, \quad \forall x \in \mathfrak{B}, \\
\nabla \rho(x)=-n_{\mathfrak{B}}(x), \quad \forall x \in \partial \mathfrak{B} .
\end{gathered}
$$

Step 3: approximating $H$ by smaller regular functions. We consider again $\varsigma$ a mollifier in $\mathbb{R}^{d}$, and we define $\varsigma_{\varepsilon}:=\varepsilon^{-d_{\varsigma}}\left(\frac{\dot{\varepsilon}}{\varepsilon}\right)$, which is supported in $B(0, \varepsilon)$ and satisfies:

$$
\int_{B(0, \varepsilon)} \varsigma_{\varepsilon}(x) d x=1 .
$$

In addition, we define in $\mathfrak{B}_{\varepsilon}$ the function $H_{\varepsilon}:=(1-\sqrt{\varepsilon}) H * \varsigma_{\varepsilon}$, which is well-defined because $x \in \mathfrak{B}_{\varepsilon}$ implies that $B(x, \varepsilon) \subset \mathfrak{B}$ (see $(A .3)$ ). Furthermore, if $x \in \mathfrak{B}_{\varepsilon}$ :

$$
\begin{aligned}
& H_{\varepsilon}(x)=(1-\sqrt{\varepsilon}) \int_{B(0, \varepsilon)} H(x-\tilde{x}) \varsigma_{\varepsilon}(\tilde{x}) d \tilde{x} \\
& \leq(1-\sqrt{\varepsilon})\left(H(x)+\varepsilon\|H\|_{W^{1, \infty}(\mathfrak{B})}\right) \int_{B(0, \varepsilon)} \varsigma_{\varepsilon}(\tilde{x}) d \tilde{x} \\
& \quad=H(x)-\sqrt{\varepsilon} H(x)+\varepsilon\|H\|_{W^{1, \infty}(\mathfrak{B})}-\varepsilon^{3 / 2}\|H\|_{W^{1, \infty}(\mathfrak{B})} .
\end{aligned}
$$

This follows from A.9 and from the fact that for all $\tilde{x} \in B(0, \varepsilon)$ we have that:

$$
H(x-\tilde{x})=H(x)-\int_{0}^{1} \nabla H(x-\tau \tilde{x}) \cdot \tilde{x} d \tau \leq H(x)+|\tilde{x}|\|H\|_{W^{1, \infty}(\mathfrak{B})} \leq H(x)+\varepsilon\|H\|_{W^{1, \infty}(\mathfrak{B})} .
$$

So, since $\inf _{\mathfrak{B}} H>0$, from A.10 we obtain for all $\varepsilon<<<\varepsilon_{0}(\Omega)$ that:

$$
H_{\varepsilon} \leq H \text { in } \mathfrak{B}_{\varepsilon}
$$

Remark A.2. We have included a term $1-\sqrt{\varepsilon}$ in the definition of $H_{\varepsilon}$ to have A.11.

Similarly, we have for all $x \in \mathfrak{B}_{\varepsilon}$ the lower bound:

$$
H_{\varepsilon}(x) \geq(1-\sqrt{\varepsilon})\left(H(x)-\varepsilon\|H\|_{W^{1, \infty}(\mathfrak{B})}\right) .
$$

Indeed, this follows from A.9 and from the fact that for all $\tilde{x} \in B(0, \varepsilon)$ we have that:

$$
H(x-\tilde{x})=H(x)-\int_{0}^{1} \nabla H(x-\tau \tilde{x}) \cdot \tilde{x} d \tau \geq H(x)-|\tilde{x}|\|H\|_{W^{1, \infty}(\mathfrak{B})} \geq H(x)-\varepsilon\|H\|_{W^{1, \infty}(\mathfrak{B})} .
$$


One important consequence of $\mathrm{A} .10$ and $\mathrm{A} .12$ is the convergence:

$$
\inf _{\mathfrak{B}_{\varepsilon}} H_{\varepsilon} \rightarrow \inf _{\mathfrak{B}} H .
$$

A last important property of the functions $H_{\varepsilon}$ is that they are uniformly Lipschitz for $\varepsilon<<<$ $\varepsilon_{0}(\Omega)$. Indeed, from Young's convolution inequality and A.9 we obtain that:

$$
\left\|\nabla H_{\varepsilon}\right\|_{L^{\infty}\left(\mathfrak{B}_{\varepsilon}\right)}=(1-\sqrt{\varepsilon})\left\|\varsigma_{\varepsilon} * \nabla H\right\|_{L^{\infty}\left(\mathfrak{B}_{\varepsilon}\right)} \leq\|\nabla H\|_{L^{\infty}(\mathfrak{B})}
$$

Step 4: approximating $\Omega$ by some regular domains $\Omega_{\varepsilon}$. We define in $\bar{\Omega}$ for all $\varepsilon$ satisfying that $\mathfrak{B}_{2 \varepsilon} \neq \emptyset$ the following functions:

$$
\mathfrak{D}_{\varepsilon}(x, z):=\chi_{\varepsilon}(x) \rho(x)\left(H_{\varepsilon}(x)-z\right) z, \quad \forall(x, z) \in \bar{\Omega},
$$

for $\chi_{\varepsilon}$ a positive $C^{\infty}\left(\mathbb{R}^{d}\right)$ cut-off function which takes values in $[0,1]$, supported in $\mathfrak{B}_{\varepsilon}$ and such that $\chi_{\varepsilon}=1$ in $\overline{\mathfrak{B}_{2 \varepsilon}}$ (for instance $1_{\mathfrak{B}_{(3 / 2) \varepsilon}} * \varsigma_{\varepsilon / 4}$ ).

Remark A.3. Since $\chi_{\varepsilon}=1$ in $\overline{\mathfrak{B}_{2 \varepsilon}}$ we obtain from $A .15$ that:

$$
\nabla \mathfrak{D}_{\varepsilon}(x, z)=\left(z \rho(x) \nabla H_{\varepsilon}(x)+z\left(H_{\varepsilon}(x)-z\right) \nabla \rho(x), \rho(x)\left(H_{\varepsilon}(x)-2 z\right)\right), \quad \forall(x, z) \in \overline{\mathfrak{B}_{2 \varepsilon}} .
$$

We approximate $\Omega$ by:

$$
\Omega_{\varepsilon}:=\mathfrak{D}_{\varepsilon}^{-1}\left(\varepsilon\|H\|_{L^{\infty}(\mathfrak{B})}^{2},+\infty\right),
$$

which is well-defined if $\varepsilon<<<\varepsilon_{0}(\Omega)$ (as in that case $\mathfrak{B}_{2 \varepsilon} \neq \emptyset$ ). We first prove (3.9). For that, we fix $\varepsilon<<<\varepsilon_{0}(\Omega)$, we also fix $(x, z) \in \Omega \backslash \Omega_{\varepsilon}$ and we get a bound of $d((x, z), \partial \Omega)$. Since $(x, z) \in \Omega \backslash \Omega_{\varepsilon}$, we have that $\mathfrak{D}_{\varepsilon}(x, z) \leq \varepsilon\|H\|_{L^{\infty}(\mathfrak{B})}^{2}$, which implies that:

$$
\min \left\{\chi_{\varepsilon}(x), \rho(x),\left(H_{\varepsilon}(x)-z\right), z\right\} \leq \varepsilon^{1 / 4}\|H\|_{L^{\infty}(\mathfrak{B})}^{2},
$$

so we are in at least one of the following cases:

- If $\chi_{\varepsilon}(x) \leq \varepsilon^{1 / 4}\|H\|_{L^{\infty}(\mathfrak{B})}^{2}$, then from $\varepsilon<<<\varepsilon_{0}(\Omega)$ we have that $x \notin \mathfrak{B}_{2 \varepsilon}$, which implies that:

$$
d((x, z), \partial \Omega) \leq \mathfrak{d}(x) \leq 2 \varepsilon
$$

- If $\rho(x) \leq \varepsilon^{1 / 4}\|H\|_{L^{\infty}(\mathfrak{B})}^{2}$, we have by A.7 that:

$$
d((x, z), \partial \Omega) \leq \mathfrak{d}(x) \leq \frac{3}{2} \varepsilon^{1 / 4}\|H\|_{L^{\infty}(\mathfrak{B})}^{2} .
$$

- If $H_{\varepsilon}(x)-z \leq \varepsilon^{1 / 4}\|H\|_{L^{\infty}(\mathfrak{B})}^{2}$, using A.10 we have that:

$$
d((x, z), \partial \Omega) \leq d((x, z), \mathfrak{T})=H(x)-z=\left(H(x)-H_{\varepsilon}(x)\right)+H_{\varepsilon}(x)-z \leq \varepsilon^{1 / 4}\|H\|_{L^{\infty}(\mathfrak{B})}^{2}+C \sqrt{\varepsilon} .
$$


- If $z \leq \varepsilon^{1 / 4}\|H\|_{L^{\infty}(\mathfrak{B})}^{2}$ we have that:

$$
d((x, z), \partial \Omega) \leq d((x, z), \mathfrak{B} \times\{0\}) \leq \varepsilon^{1 / 4}\|H\|_{L^{\infty}(\mathfrak{B})}^{2} .
$$

So, from A.17)-A.20 we obtain for all $\varepsilon<<<\varepsilon_{0}(\Omega)$ and $(x, z) \in \Omega \backslash \Omega_{\varepsilon}$ that:

$$
d((x, z), \partial \Omega) \leq C \varepsilon^{1 / 4}
$$

which implies 3.9 .

Step 5: proof of the regularity of the domains $\Omega_{\varepsilon}$. Next, let us prove that if $\varepsilon<<<\varepsilon_{0}(\Omega, \omega)$ the open sets $\Omega_{\varepsilon}$ satisfy (2.7) and (2.8). Indeed, if $\varepsilon<<<\varepsilon_{0}(\Omega, \omega)$ we have by (3.9) that:

$$
\sup _{\Omega \backslash \Omega_{\varepsilon}} d(\cdot, \partial \Omega)<d(\omega, \partial \Omega),
$$

which implies that $\omega \subset \Omega_{\varepsilon}$. Consequently, we only have to prove that if $\varepsilon<<<\varepsilon_{0}(\Omega)$ the domains $\Omega_{\varepsilon}$ are $C^{2}$ (see Remark 2.9), which we will prove by using Lemma A.1. So we fix $\varepsilon<<<\varepsilon_{0}(\Omega)$ and check that the domain $\Omega_{\varepsilon}$ satisfies the requirements of Lemma A.1.

First, it is clear that $\mathfrak{D}_{\varepsilon}$ is a function which belongs to $C^{2}(\bar{\Omega})\left(\rho \in C^{2}\left(\operatorname{supp}\left(\chi_{\varepsilon}\right)\right)\right.$ by A.5) . Moreover, it satisfies $\mathfrak{D}_{\varepsilon} \leq 0$ on $\partial \Omega$. Indeed, we consider A.15 and the division of the boundary as stated in Remark 1.2:

- If $(x, z) \in \mathfrak{B} \times\{0\}$, then $\mathfrak{D}_{\varepsilon}(x, z)=0$.

- If $(x, z) \in \mathfrak{L}$, then $x \in \partial \mathfrak{B}$, so $\chi_{\varepsilon}(x)=0$ (by the support of $\chi_{\varepsilon}$ ), so $\mathfrak{D}_{\varepsilon}(x, z)=0$.

- If $(x, z) \in \mathfrak{T}$, then $z=H(x)$. If $\mathfrak{D}_{\varepsilon}(x, z) \neq 0$ we have that $x \in \operatorname{supp}\left(\chi_{\varepsilon}\right) \subset \mathfrak{B}_{\varepsilon}$, so $H_{\varepsilon}(x) \leq H(x)$ by A.11, so $\mathfrak{D}_{\varepsilon}(x, z)<0$; otherwise, $\mathfrak{D}_{\varepsilon}(x, z)=0$. Consequently, if $(x, z) \in \mathfrak{T}$ we have $\mathfrak{D}_{\varepsilon}(x, z) \leq 0$.

Thus, to apply Lemma A.1 it remains to check that:

$$
\mathfrak{D}_{\varepsilon}^{-1}\left(\varepsilon\|H\|_{L^{\infty}(\Omega)}^{2}\right) \cap\left(\nabla \mathfrak{D}_{\varepsilon}\right)^{-1}(\{0\})=\emptyset .
$$

For that purpose we fix $(x, z) \in \Omega$ satisfying:

$$
\mathfrak{D}_{\varepsilon}(x, z)=\varepsilon\|H\|_{L^{\infty}(\mathfrak{B})}^{2}, \quad \partial_{z} \mathfrak{D}_{\varepsilon}(x, z)=0,
$$

and prove that $\nabla_{x} \mathfrak{D}_{\varepsilon}(x, z) \neq 0$. 
We first remark that from $\mathrm{A} .22{ }_{1}$ we have that:

$$
x \in \overline{\mathfrak{B}_{2 \varepsilon}} .
$$

Indeed, from A.11 we obtain that:

$$
\chi_{\varepsilon}(x)\left(H_{\varepsilon}(x)-z\right) z \leq \frac{\|H\|_{L^{\infty}(\mathfrak{B})}^{2}}{4},
$$

which implies by A.22 that:

$$
\rho(x) \geq 4 \varepsilon,
$$

which implies using A.7 that:

$$
\mathfrak{d}(x) \geq 2 \varepsilon,
$$

that is, A.23.

From A.16 and A.22 ${ }_{2}$ we get that $z=H_{\varepsilon}(x) / 2$. Hence, from A.23 and A.22 ${ }_{1}$ we have the equality:

$$
\rho(x)=\frac{4 \varepsilon\|H\|_{L^{\infty}(\mathfrak{B})}^{2}}{H_{\varepsilon}^{2}(x)},
$$

which implies together with A.16 the equality:

$$
\nabla \mathfrak{D}_{\varepsilon}(x, z)=\left(\frac{2 \varepsilon\|H\|_{L^{\infty}(\mathfrak{B})}^{2}}{H_{\varepsilon}(x)} \nabla H_{\varepsilon}(x)+\frac{H_{\varepsilon}^{2}(x)}{4} \nabla \rho(x), 0\right) .
$$

So, because of A.13 and A.14 the previous equality turns into:

$$
\nabla \mathfrak{D}_{\varepsilon}(x, z)=\left(O(\varepsilon)+\frac{H_{\varepsilon}^{2}(x)}{4} \nabla \rho(x), 0\right) .
$$

So, from A.26, A.25, $\varepsilon<<<\varepsilon_{0}(\Omega)$, A.13, A.5, A.7 and A.8 we obtain that A.22 implies that $\nabla_{x} \mathfrak{D}_{\varepsilon}(x, z) \neq 0$, which proves A.21.

Consequently, thanks to Lemma A.1, if $\varepsilon<<<\varepsilon_{0}(\Omega)$, we have that $\Omega_{\varepsilon}$ is $C^{2}$ and:

$$
\partial \Omega_{\varepsilon}=\mathfrak{D}_{\varepsilon}^{-1}\left(\varepsilon\|H\|_{L^{\infty}(\mathfrak{B})}^{2}\right) .
$$

Remark A.4. In order to prove A.23 we have only used A.22 ${ }_{1}$ and that $\varepsilon<<<\varepsilon_{0}(\Omega)$. Consequently, if $\varepsilon<<<\varepsilon_{0}(\Omega)$, considering (A.27), A.23) and that $\mathfrak{B}_{2 \varepsilon} \subset \chi_{\varepsilon}^{-1}(\{1\})$ we find that:

$$
\rho(x)\left(H_{\varepsilon}(x)-z\right) z=\varepsilon\|H\|_{L^{\infty}(\mathfrak{B})}^{2}, \quad \forall(x, z) \in \partial \Omega_{\varepsilon} .
$$

Similarly, if $\varepsilon<<<\varepsilon_{0}(\Omega)$ and $(x, z) \in \partial \Omega_{\varepsilon}$, using (A.2, A.16) and A.23) we obtain that:

$$
n_{\varepsilon}(x, z)=-\frac{\nabla \mathfrak{D}_{\varepsilon}(x, z)}{\left|\nabla \mathfrak{D}_{\varepsilon}(x, z)\right|}=\frac{\left(-z \rho(x) \nabla H_{\varepsilon}(x)-z\left(H_{\varepsilon}(x)-z\right) \nabla \rho(x), \rho(x)\left(2 z-H_{\varepsilon}(x)\right)\right)}{\left|\left(-z \rho(x) \nabla H_{\varepsilon}(x)-z\left(H_{\varepsilon}(x)-z\right) \nabla \rho(x), \rho(x)\left(2 z-H_{\varepsilon}(x)\right)\right)\right|},
$$

where $n_{\varepsilon}$ denotes the outward unit normal vector on $\partial \Omega_{\varepsilon}$. 
Step 6: the domains $\Omega_{\varepsilon}$ satisfy 2.9. Considering (A.29) and (3.5), we have to prove that if $\mathfrak{c}>>>\mathfrak{c}_{0}\left(\Omega, \omega, \eta_{\mathfrak{B}}\right), \varepsilon<<<\varepsilon_{0}\left(\Omega, \omega, \eta_{\mathfrak{B}}, \mathfrak{c}\right)$ and $(x, z) \in \partial \Omega_{\varepsilon}$, we have that:

$$
-z \rho(x) \nabla H_{\varepsilon}(x) \cdot \nabla \eta_{\mathfrak{B}}(x)-z\left(H_{\varepsilon}(x)-z\right) \nabla \rho(x) \cdot \nabla \eta_{\mathfrak{B}}(x)-2 \rho(x)\left(2 z-H_{\varepsilon}(x)\right) \mathfrak{c}(z-\bar{z}) \leq 0 .
$$

Intuitively, the proof consists on using that $\partial \Omega_{\varepsilon}$ is near $\partial \Omega$ and that $n_{\varepsilon}$ somehow approximates $n$. Indeed, we follow different (but a finite number of) approaches depending if we are close to $\mathfrak{B} \times\{0\}, \mathfrak{L}$ or $\mathfrak{T}$ (see Remark 1.2 for the notation). This is necessary because $\eta$ and $n_{\varepsilon}$ have different behaviours in different parts of the boundary (they do not present any symmetry), so considering only A.24 does not suffice and we have to be more precise.

Rigorously, we fix first $\mathfrak{c} \geq \mathfrak{c}_{o}\left(\Omega, \omega, \eta_{\mathfrak{B}}\right)$, second $\varepsilon<<<\varepsilon_{0}\left(\Omega, \omega, \eta_{\mathfrak{B}}, \mathfrak{c}\right)$ and third $(x, z) \in \partial \Omega_{\varepsilon}$, for $\mathfrak{c}_{0}$ and $\varepsilon_{0}$ to be obtained during the proof. We prove A.30 for those values. As stated before, we follow different (but a finite number of) approaches depending on $(x, z)$. A first partition of the boundary is the following:

$$
\left\{\begin{array}{l}
\partial \Omega_{\varepsilon, b}:=\partial \Omega_{\varepsilon} \cap\left\{(x, z): z \in\left(0, H_{\varepsilon}(x) / 2\right]\right\}, \\
\partial \Omega_{\varepsilon, t}:=\partial \Omega_{\varepsilon} \cap\left\{(x, z): z \in\left(H_{\varepsilon}(x) / 2, H_{\varepsilon}(x)\right)\right\} .
\end{array}\right.
$$

Case 1: if $(x, z) \in \partial \Omega_{\varepsilon, b}$. From $\varepsilon<<<\varepsilon_{0}(\Omega)$, A.31 ${ }_{1}$, A.28), and A.11 we obtain that:

$$
\varepsilon\|H\|_{L^{\infty}(\mathfrak{B})} \leq z \rho(x) \leq \frac{2 \varepsilon\|H\|_{L^{\infty}(\mathfrak{B})}^{2}}{\inf _{\mathfrak{B}_{\varepsilon}} H_{\varepsilon}} .
$$

Thanks to A.13), from $\varepsilon<<<\varepsilon_{0}(\Omega)$ we have the inequality:

$$
\frac{1}{\inf _{\mathfrak{B}_{\varepsilon}} H_{\varepsilon}} \leq \frac{2}{\inf _{\mathfrak{B}} H}
$$

so if we define:

$$
\kappa_{1}:=\|H\|_{L^{\infty}(\mathfrak{B})}, \quad \kappa_{2}:=\frac{4\|H\|_{L^{\infty}(\mathfrak{B})}^{2}}{\inf _{\mathfrak{B}} H},
$$

we have that $\mathrm{A} .32$ and $\varepsilon<<<\varepsilon_{0}(\Omega)$ imply that:

$$
\kappa_{1} \varepsilon \leq z \rho(x) \leq \kappa_{2} \varepsilon
$$

So, either $\rho(x) \leq \sqrt{\kappa_{2} \varepsilon}$ or $z \leq \sqrt{\kappa_{2} \varepsilon}$ is obtained. We study both cases separately, since in the first one $(x, z)$ is near $\mathfrak{L}$, whereas in the second one $(x, z)$ is near $\mathfrak{B} \times\{0\}$. 
Case 1.1: if $(x, z) \in \partial \Omega_{\varepsilon, b}$ and if $\rho(x) \leq \sqrt{\kappa_{2} \varepsilon}$. Due to A.34 we have the bound:

$$
z \geq \kappa_{1} \sqrt{\kappa_{2}^{-1} \varepsilon}
$$

We first focus on the sign of:

$$
-z \rho(x) \nabla H_{\varepsilon}(x) \cdot \nabla \eta_{\mathfrak{B}}(x)-z\left(H_{\varepsilon}(x)-z\right) \nabla \rho(x) \cdot \nabla \eta_{\mathfrak{B}}(x) .
$$

From A.34 we obtain the estimate:

$$
\left|z \rho(x) \nabla H_{\varepsilon}(x) \cdot \nabla \eta_{\mathfrak{B}}(x)\right| \leq C \varepsilon
$$

Moreover, we have the equality (see A.4):

$$
-z\left(H_{\varepsilon}(x)-z\right) \nabla \rho(x)=z\left(H_{\varepsilon}(x)-z\right) n_{\mathfrak{B}}(P(x))+z\left(H_{\varepsilon}(x)-z\right)\left(-n_{\mathfrak{B}}(P(x))-\nabla \rho(x)\right) .
$$

Recalling A.4 - A.8, from $\varepsilon<<<\varepsilon_{0}(\Omega)$ we obtain the estimate:

$$
\begin{aligned}
\left|-n_{\mathfrak{B}}(P(x))-\nabla \rho(x)\right| & =|\nabla \rho(P(x))-\nabla \rho(x)| \\
\leq & \|\rho\|_{W^{2, \infty}(\mathfrak{B})}|P(x)-x|=\|\rho\|_{W^{2, \infty}(\mathfrak{B})} \mathfrak{d}(x) \leq \frac{3}{2}\|\rho\|_{W^{2, \infty}(\mathfrak{B})} \rho(x) .
\end{aligned}
$$

So, combining A.38 and A.34 we get the bound:

$$
\left|z\left(H_{\varepsilon}(x)-z\right)\left(-n_{\mathfrak{B}}(P(x))-\nabla \rho(x)\right) \cdot \nabla \eta_{\mathfrak{B}}(x)\right| \leq C|z \rho(x)| \leq C \varepsilon
$$

Finally, we have the equality:

$$
\begin{aligned}
z\left(H_{\varepsilon}(x)-z\right) n_{\mathfrak{B}}(P(x)) \cdot \nabla \eta_{\mathfrak{B}}(x) & =z\left(H_{\varepsilon}(x)-z\right) n_{\mathfrak{B}}(P(x)) \cdot \nabla \eta_{\mathfrak{B}}(P(x)) \\
+ & z\left(H_{\varepsilon}(x)-z\right) n_{\mathfrak{B}}(P(x)) \cdot\left(-\nabla \eta_{\mathfrak{B}}(P(x))+\nabla \eta_{\mathfrak{B}}(x)\right) .
\end{aligned}
$$

Since $\eta_{\mathfrak{B}} \in C^{2}(\overline{\mathfrak{B}})$, arguing similarly to A.38 we obtain the estimate:

$$
\left|z\left(H_{\varepsilon}(x)-z\right) n_{\mathfrak{B}}(P(x)) \cdot\left(-\nabla \eta_{\mathfrak{B}}(P(x))+\nabla \eta_{\mathfrak{B}}(x)\right)\right| \leq C \varepsilon
$$

Moreover, if we take into account A.35, A.31, A.13 and 3.4 we have the bound:

$$
z\left(H_{\varepsilon}(x)-z\right) n_{\mathfrak{B}}(P(x)) \cdot \nabla \eta_{\mathfrak{B}}(P(x)) \leq-c \sqrt{\varepsilon} .
$$

Consequently, if we combine A.36- A.42, we find that:

$$
-z \rho(x) \nabla H_{\varepsilon}(x) \cdot \nabla \eta_{\mathfrak{B}}(x)-z\left(H_{\varepsilon}(x)-z\right) \nabla \rho(x) \cdot \nabla \eta_{\mathfrak{B}}(x) \leq C \varepsilon-c \sqrt{\varepsilon} .
$$


Let us now analyze the term:

$$
-2 \rho(x)\left(2 z-H_{\varepsilon}(x)\right) \mathfrak{c}(z-\bar{z})=2 \rho(x)\left(H_{\varepsilon}(x)-2 z\right) \mathfrak{c}(z-\bar{z}) .
$$

- On the one hand, if $z \leq \bar{z}$ (see $(3.1)$ for the definition of $\bar{z}$ ), we have that (see $\left.(A .31)_{1}\right)$ :

$$
2 \rho(x)\left(H_{\varepsilon}(x)-2 z\right) \mathfrak{c}(z-\bar{z}) \leq 0,
$$

which, together with A.43 implies A.30 since $\varepsilon<<<\varepsilon_{0}\left(\Omega, \omega, \eta_{\mathfrak{B}}\right)$.

- On the other hand, if $z \geq \bar{z}$, we get from A.34 the inequality:

$$
\rho(x) \leq C \varepsilon
$$

Thus, we obtain the estimate:

$$
\left|2 \rho(x)\left(H_{\varepsilon}(x)-2 z\right) \mathfrak{c}(z-\bar{z})\right| \leq C \mathfrak{c} \varepsilon .
$$

Therefore, if we combine A.43 and A.45, we get the bound:

$$
\begin{array}{r}
-z \rho(x) \nabla H_{\varepsilon}(x) \cdot \nabla \eta_{\mathfrak{B}}(x)-z\left(H_{\varepsilon}(x)-z\right) \nabla \rho(x) \cdot \nabla \eta_{\mathfrak{B}}(x)-2 \rho(x)\left(2 z-H_{\varepsilon}(x)\right) \mathfrak{c}(z-\bar{z}) \\
\leq C(1+\mathfrak{c}) \varepsilon-c \sqrt{\varepsilon}
\end{array}
$$

which implies A.30 since $\varepsilon<<<\varepsilon_{0}\left(\Omega, \omega, \eta_{\mathfrak{B}}, \mathfrak{c}\right)$.

Remark A.5. Note that for some control domains there are $(x, z) \in \partial \Omega_{\varepsilon, b}$ satisfying $\rho(x) \leq \sqrt{\kappa_{2} \varepsilon}$ and A.44. In fact, A.44 provides us some additional information because $\sqrt{\varepsilon}>>>\varepsilon$ for $\varepsilon$ small. Moreover, this does not suppose a contradiction with A.24 because it just means that $C>4$ in $\mathrm{A} .44$.

Case 1.2: if $(x, z) \in \partial \Omega_{\varepsilon, b}$ and if $z \leq \sqrt{\kappa_{2} \varepsilon}$. We first remark that $\varepsilon<<<\varepsilon_{0}(\Omega, \omega)$ implies:

$$
z \leq \sqrt{\kappa_{2} \varepsilon} \leq \frac{\bar{z}}{4} \leq \frac{\inf _{\mathfrak{B}} H}{4}
$$

This can be done because $\kappa_{1}, \kappa_{2}$ and $\bar{z}$ are strictly positive constants that only depend on $\Omega$ and $\omega$ (see A.33) and (3.1). Moreover, we have from A.34 that:

$$
\rho(x) \geq \kappa_{2} \sqrt{\kappa_{1}^{-1} \varepsilon}
$$

If we use A.34, A.14, A.11, that $z \leq \sqrt{\kappa_{2} \varepsilon}$, A.5, that $\eta_{\mathfrak{B}} \in C^{2}(\overline{\mathfrak{B}})$, A.47, A.46 and A.13, we obtain from $\varepsilon<<<\varepsilon_{0}(\Omega, \omega)$ the inequality:

$$
\begin{aligned}
-z \rho(x) \nabla H_{\varepsilon}(x) \cdot \nabla \eta_{\mathfrak{B}}(x)-z\left(H_{\varepsilon}(x)-z\right) \nabla \rho(x) \cdot \nabla \eta_{\mathfrak{B}}(x)-2 \rho(x)(2 z & \left.-H_{\varepsilon}(x)\right) \mathfrak{c}(z-\bar{z}) \\
& \leq C \varepsilon+C \varepsilon^{1 / 2}-c \mathfrak{c} \varepsilon^{1 / 2}
\end{aligned}
$$

Consequently, as $\mathfrak{c}>>>\mathfrak{c}_{0}\left(\Omega, \omega, \eta_{\mathfrak{B}}\right)$ and $\varepsilon<<<\varepsilon_{0}\left(\Omega, \omega, \eta_{\mathfrak{B}}, \mathfrak{c}\right)$, we have A.30. 
Case 2: if $(x, z) \in \partial \Omega_{\varepsilon, t}$. As for $\partial \Omega_{\varepsilon, t}$ the situation is very similar. Indeed, from A.28 we find the bounds:

$$
\kappa_{1} \varepsilon \leq\left(H_{\varepsilon}(x)-z\right) \rho(x) \leq \kappa_{2} \varepsilon,
$$

for $\kappa_{1}$ and $\kappa_{2}$ defined in A.33. As before, we distinguish the cases $\rho(x) \leq \sqrt{\kappa_{2} \varepsilon}$ and $H_{\varepsilon}(x)-z \leq$ $\sqrt{\kappa_{2} \varepsilon}$. The first one concerns the points near $\mathfrak{L}$, whereas the second one concerns the points near $\mathfrak{T}$.

Case 2.1: if $(x, z) \in \partial \Omega_{\varepsilon, t}$ and if $\rho(x) \leq \sqrt{\kappa_{2} \varepsilon}$. Due to A.48 we have the bound:

$$
H_{\varepsilon}(x)-z \geq \kappa_{1} \sqrt{\kappa_{2}^{-1} \varepsilon} .
$$

Arguing as in the case 1.1, we get the estimate:

$$
-z\left(H_{\varepsilon}(x)-z\right) \nabla \rho(x) \cdot \nabla \eta_{\mathfrak{B}}(x) \leq C \varepsilon-c \varepsilon^{1 / 2} .
$$

In order to continue, as before, we make a distinction depending on how close $z$ is to $H_{\varepsilon}(x)$ :

- We start with the subcase:

$$
z \geq \max \left\{\sqrt{\frac{\bar{z}}{\inf _{\mathfrak{B}} H}}, \frac{2}{3}\right\} H_{\varepsilon}(x) .
$$

We obtain from $(3.2)$ and $\varepsilon<<<\varepsilon_{0}(\Omega, \omega)$ the inequality:

$$
H_{\varepsilon}(x) \geq \sqrt[4]{\frac{\bar{z}}{\inf _{\mathfrak{B}} H}} H(x)
$$

Thanks to A.51, A.52 and $\varepsilon<<<\varepsilon_{0}(\Omega, \omega)$, we have the lower bound:

$$
z \geq \sqrt[4]{(\bar{z})^{3} \inf _{\mathfrak{B}} H}=\sqrt[4]{\frac{\inf _{\mathfrak{B}} H}{\bar{z}}} \bar{z} .
$$

So, combining A.51, A.13, A.53) and (3.2) and that $\varepsilon<<<\varepsilon_{0}(\Omega, \omega)$, we obtain the inequality:

$$
-2 \rho(x)\left(2 z-H_{\varepsilon}(x)\right) \mathfrak{c}(z-\bar{z}) \leq-c \mathfrak{c} \rho(x) .
$$

Moreover, we clearly have the estimate:

$$
-z \rho(x) \nabla H_{\varepsilon}(x) \cdot \nabla \eta_{\mathfrak{B}}(x) \leq C \rho(x) .
$$

Thus, we get from $\mathfrak{c}>>>\mathfrak{c}_{0}\left(\Omega, \omega, \eta_{\mathfrak{B}}\right), \rho \geq 0$, A.54 and A.55 the upper bound:

$$
-z \rho(x) \nabla H_{\varepsilon}(x) \cdot \nabla \eta_{\mathfrak{B}}(x)-2 \rho(x)\left(2 z-H_{\varepsilon}(x)\right) \mathfrak{c}(z-\bar{z}) \leq 0
$$

Hence, combining A.50 and A.56 we get A.30 from $\varepsilon<<<\varepsilon_{0}\left(\Omega, \omega, \eta_{\mathfrak{B}}\right)$. 
- Let us now suppose that:

$$
z<\max \left\{\sqrt{\frac{\bar{z}}{\inf _{\mathfrak{B}} H}}, \frac{2}{3}\right\} H_{\varepsilon}(x) .
$$

Considering A.48 and A.13 we have that:

$$
\rho(x)<C \varepsilon
$$

Thus, we find the estimate:

$$
-z \rho(x) \nabla H_{\varepsilon}(x) \cdot \nabla \eta_{\mathfrak{B}}(x)-2 \rho(x)\left(2 z-H_{\varepsilon}(x)\right) \mathfrak{c}(z-\bar{z})<C \mathfrak{c} \varepsilon .
$$

So, from A.50, A.59 and $\varepsilon<<<\varepsilon_{0}\left(\Omega, \omega, \eta_{\mathfrak{B}}, \mathfrak{c}\right)$ we obtain A.30 .

Remark A.6. Again, note that for some control domains there are $(x, z) \in \partial \Omega_{\varepsilon, t}$ satisfying $\rho(x) \leq \sqrt{\kappa_{2} \varepsilon}$ and A.58. In fact, A.58 provides us some additional information because $\sqrt{\varepsilon}>>>$ for $\varepsilon$ small. Moreover, this does not suppose a contradiction with A.24 because it just means that $C>4$ in $\mathrm{A} .58$.

Case 2.2: if $(x, z) \in \partial \Omega_{\varepsilon, t}$ and if $H_{\varepsilon}(x)-z \leq \sqrt{\kappa_{2} \varepsilon}$. We have from (A.48) the inequality:

$$
\rho(x) \geq \kappa_{1} \sqrt{\kappa_{2}^{-1} \varepsilon} .
$$

Moreover, from A.14, $H_{\varepsilon}(x)-z \leq \sqrt{\kappa_{2} \varepsilon}$ and A.60 we find the upper bound:

$$
-z \rho(x) \nabla H_{\varepsilon}(x) \cdot \nabla \eta_{\mathfrak{B}}(x)-z\left(H_{\varepsilon}(x)-z\right) \nabla \rho(x) \cdot \nabla \eta_{\mathfrak{B}}(x) \leq C\left(\rho(x)+\varepsilon^{1 / 2}\right) \leq C \rho(x) .
$$

In addition, since $H_{\varepsilon}(x)-z \leq \sqrt{\kappa_{2} \varepsilon}$ and $\varepsilon<<<\varepsilon_{0}(\Omega, \omega)$ we have that:

$$
z>\max \left\{\sqrt{\frac{\inf _{\mathfrak{B}_{\varepsilon}} H_{\varepsilon}}{\bar{z}}} \bar{z}, \frac{2}{3} H_{\varepsilon}(x)\right\} .
$$

Indeed, because of $\mathrm{A} .13), H_{\varepsilon}(x)-z \leq \sqrt{\kappa_{2} \varepsilon}$ and $\varepsilon<<<\varepsilon_{0}(\Omega)$ we have that:

$$
z \geq H_{\varepsilon}(x)-\sqrt{\kappa_{2} \varepsilon} \geq H_{\varepsilon}(x)-\frac{1}{6} \inf _{\mathfrak{B}} H \geq H_{\varepsilon}(x)-\frac{1}{3} \inf _{\mathfrak{B}_{\varepsilon}} H_{\varepsilon}(x) \geq \frac{2}{3} H_{\varepsilon}(x) .
$$

Similarly, as a consequence of A.13, 3.2 and $\varepsilon<<<\varepsilon_{0}(\Omega, \omega)$ we have that:

$$
\begin{aligned}
z \geq H_{\varepsilon}(x)-\sqrt{\kappa_{2} \varepsilon} & \geq \inf _{\mathfrak{B}_{\varepsilon}} H_{\varepsilon}-\sqrt{\kappa_{2} \varepsilon} \geq \inf _{\mathfrak{B}_{\varepsilon}} H_{\varepsilon}-\frac{1}{2}\left(1-\sqrt{\left.\frac{\bar{z}}{\inf _{\mathfrak{B}} H}\right)} \inf _{\mathfrak{B}} H\right. \\
& \geq \inf _{\mathfrak{B}_{\varepsilon}} H_{\varepsilon}-\left(1-\sqrt{\frac{\bar{z}}{\inf _{\mathfrak{B}_{\varepsilon}} H_{\varepsilon}}}\right) \inf _{\mathfrak{B}_{\varepsilon}} H_{\varepsilon}=\sqrt{\frac{\bar{z}}{\inf _{\mathfrak{B}_{\varepsilon}} H_{\varepsilon}}} \inf _{\mathfrak{B}_{\varepsilon}} H_{\varepsilon}=\sqrt{\frac{\inf _{\mathfrak{B}_{\varepsilon}} H_{\varepsilon}}{\bar{z}} \bar{z}}
\end{aligned}
$$

So, from A.62, A.13 and 3.2 we find that:

$$
-2 \rho(x)\left(2 z-H_{\varepsilon}(x)\right) \mathfrak{c}(z-\bar{z}) \leq-c \mathfrak{c} \rho(x) .
$$

Consequently, from A.61, A.63) and $\mathfrak{c}>>>\mathfrak{c}_{0}\left(\Omega, \omega, \eta_{\mathfrak{B}}\right)$ we obtain A.30. 


\section{B The existence of a pseudo-cylinder which is not locally star- shaped}

We show in this section the existence of a pseudo-cylinder in $\mathbb{R}^{2}$ which is not locally starshaped. The structure of this section is the following: first we recall the definition of locally star-shaped as stated in [3], but adapted to our notation, second we construct the pseudocylinder, and thirdly we prove that it is not locally star-shaped.

Definition B.1. A Lipschitz domain $\Omega$ is locally star-shaped if for all $p \in \partial \Omega$ there is $r_{p}>0$ and $q_{p} \in \Omega$ such that $\left|p-q_{p}\right|<r_{p}$ and $B\left(q_{p}, r_{p}\right) \cap \Omega$ is star-shaped with center in $q_{p}$.

Remark B.2. As a consequence of [3, Remark 3.5] we can choose $r_{p}$ as small as needed. This implies that a locally star-shaped domain can also be defined as a Lipschitz domain $\Omega$ such that for all $p \in \partial \Omega$ and all $r>0$ there is some $\tilde{r} \in(0, r)$ and $q \in \Omega$ satisfying $|p-q|<\tilde{r}$ and that $B(q, \tilde{r}) \cap \Omega$ is star-shaped with center in $q$.

We define:

$$
\Omega=\{(x, z): x \in(0,1), z \in(\tilde{H}(x), 3)\}
$$

for

$$
\tilde{H}(x):=6 \int_{0}^{x} h(s) d s, \quad \text { for } h(x):=\sum_{i \in \mathbb{N}_{*}} 1_{\left[1 / 3^{i}, 2 / 3^{i}\right)}(x)-\sum_{i \in \mathbb{N}_{*}} 1_{\left[2 / 3^{i}, 1 / 3^{i-1}\right)}(x) .
$$

We have that $\Omega$ clearly is a pseudo-cylinder (take $U(x, z)=(x, 3-z), \mathfrak{B}=(0,1)$ and $H(x)=$ $3-\tilde{H}(x)$ in Definition 1.1, as $\left.\inf _{(0,1)} H(x)=H(2 / 3)=3-2=1\right)$. See Figure 5 for an approximate illustration of $\Omega$ near each point $\left(\frac{2}{3^{i+2}}, \frac{2}{3^{i+1}}\right)$, for $i \in \mathbb{N}_{*}$.

Remark B.3. $\tilde{H}$ is a positive Lipschitz function which satisfies for all $i \in \mathbb{N}_{*}$ that $\tilde{H}(x)=$ $-\frac{2}{3^{i-1}}+6 x$ in $\left[1 / 3^{i}, 2 / 3^{i}\right)$ and:

$$
\tilde{H}(x)=\frac{2}{3^{i-2}}-6 x, \quad \forall x \in\left[2 / 3^{i}, 1 / 3^{i-1}\right) .
$$

Thus, its local minimums are taken at $x=0$ and $x=3^{-i}$ for all $i \in \mathbb{N}$ (with $\tilde{H}\left(3^{-i}\right)=0$ ). In addition, its local maximums are taken at $x=2 \cdot 3^{-i}$, for all $i \in \mathbb{N}_{*}\left(\right.$ with $\tilde{H}\left(2 \cdot 3^{-i}\right)=3 \cdot\left(2 \cdot 3^{-i}\right)$ ). In fact, $\tilde{H}(x) \leq 3 x$ for all $x \in[0,1]$, being equal if and only if $x=0$ or $x=2 \cdot 3^{-i}$, for all $i \in \mathbb{N}_{*}$.

In order to prove that $\Omega$ is not locally star-shaped we show that for all $q \in B(0,1 / 3) \cap \Omega$ there is a segment $\ell$ of length $|q|$ with one end in $q$ and such that $\ell \cap \Omega$ is not connected. This shows that the alternative definition given in Remark B.2 is not satisfied for $p=(0,0)$ and $r=1 / 3$. Hence, we fix $q=\left(x_{q}, z_{q}\right) \in B(0,1 / 3) \cap \Omega$ and follow different approaches depending on $q$ : 


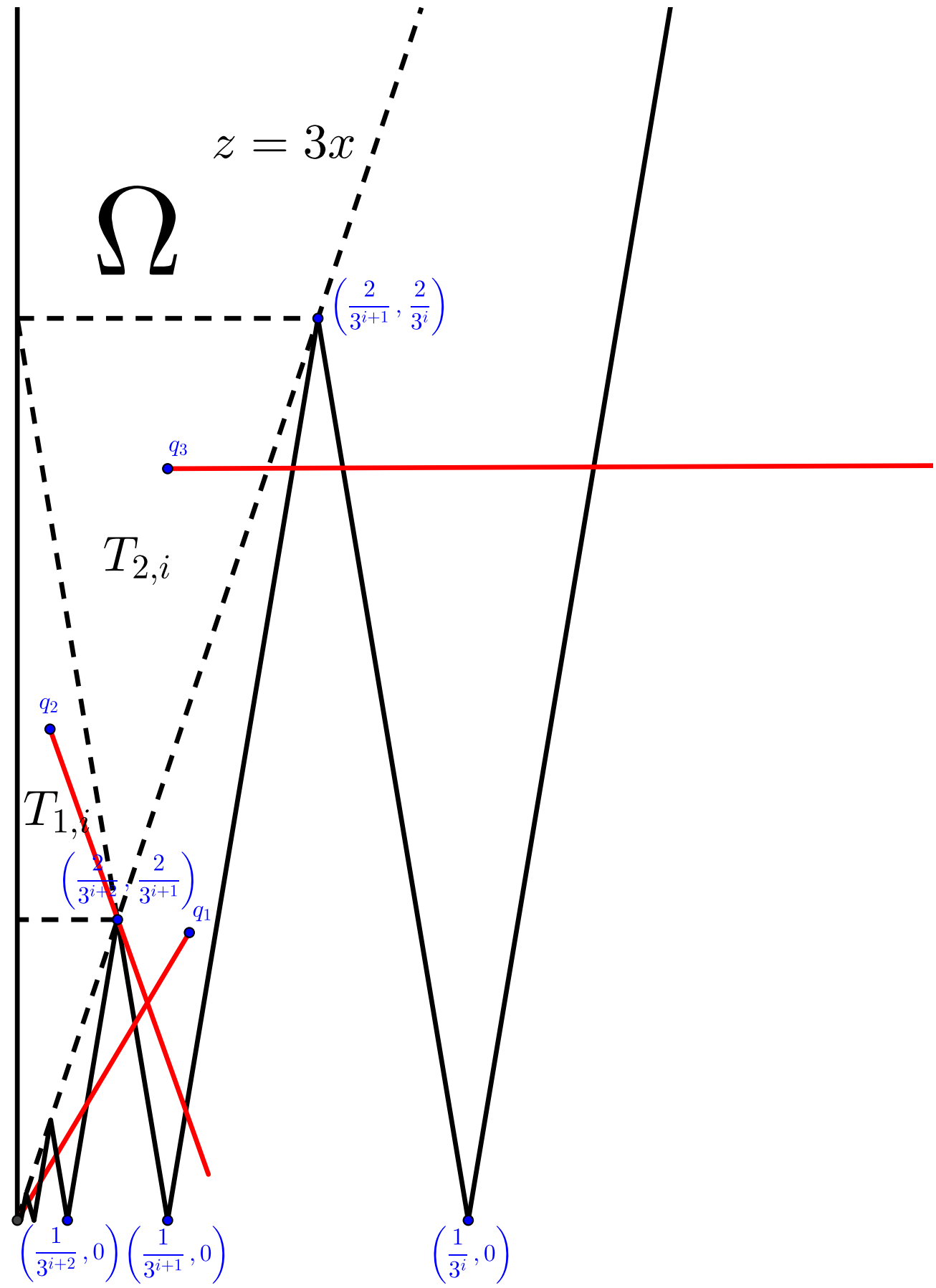

Figure 5: A pseudo-cylinder which is not locally star-shaped (approximate figure). 
Case 1: if $z_{q}<3 x_{q}$ (see $q_{1}$ in Figure 5). If $z_{q}<3 x_{q}$, we consider $\left(x_{q}>0\right.$ because $\left.q \in \Omega\right)$ :

$$
\ell=\left\{\left(x, \frac{z_{q}}{x_{q}} x\right): x \in\left[0, x_{q}\right]\right\}
$$

Clearly, $\ell$ is of length $|q|$ and one of its ends is $q$. In addition, we have that $\ell$ leaves and enters $\Omega$ an infinite number of times, as we have for all $i \in \mathbb{N}_{*}$ that:

$$
\frac{z_{q}}{x_{q}} \cdot\left(3^{-i}\right)>0=\tilde{H}\left(3^{-i}\right), \quad \frac{z_{q}}{x_{q}} \cdot\left(2 \cdot 3^{-i}\right)<3 \cdot\left(2 \cdot 3^{-i}\right)=\tilde{H}\left(2 \cdot 3^{-i}\right)
$$

so $\ell \cap \Omega$ is not connected.

Case 2: if $z_{q} \geq 3 x_{q}$ and if there is $i \in \mathbb{N}_{*}$ such that $z_{q} \in\left[2 / 3^{i+1}, 2 / 3^{i}\right.$ ) and $z_{q}<\frac{2}{3^{i}}-6 x_{q}$ (see $q_{2}$ in Figure 5). Under these hypotheses the point $q$ is in the following triangle:

$$
T_{1, i}:=\left\{(x, z) \in \mathbb{R}^{2}: z \geq \frac{2}{3^{i+1}}, x>0, z<\frac{2}{3^{i}}-6 x\right\},
$$

whose vertices are:

$$
\left\{\left(0, \frac{2}{3^{i+1}}\right),\left(\frac{2}{3^{i+2}}, \frac{2}{3^{i+1}}\right),\left(0, \frac{2}{3^{i}}\right)\right\} .
$$

A consequence of $(\overline{B .2})$ is that:

$$
T_{1, i} \subset\left\{(x, z): x<\frac{2}{3^{i+2}}\right\}
$$

Let us first prove that:

$$
d\left(q,\left(\frac{2}{3^{i+2}}, \frac{2}{3^{i+1}}\right)\right)<|q| .
$$

We remark that for all $(x, z) \in \overline{T_{1, i}}$ we have that:

$$
\begin{aligned}
d^{2}\left((x, z),\left(\frac{2}{3^{i+2}},\right.\right. & \left.\left.\frac{2}{3^{i+1}}\right)\right)=\left(\frac{2}{3^{i+2}}-x\right)^{2}+\left(z-\frac{2}{3^{i+1}}\right)^{2} \\
& =x^{2}+z^{2}-\frac{4}{3^{i+2}} x-\frac{4}{3^{i+1}} z+\frac{4}{3^{2 i+4}}+\frac{4}{3^{2 i+2}}:=|(x, z)|^{2}+\mathcal{G}_{1}(x, z) .
\end{aligned}
$$

Therefore, proving (B.5) reduces to finding the maximum of $\mathcal{G}_{1}$ on $\overline{T_{1, i}}$, which is a simplex. Since $\mathcal{G}_{1}$ is an affine function, it is well-known that the maximum is reached on the vertices, which in this case are given by (B.3. Since:

$$
\mathcal{G}_{1}\left(0, \frac{2}{3^{i+1}}\right)=-\frac{32}{3^{2 i+4}}, \quad \mathcal{G}_{1}\left(\frac{2}{3^{i+2}}, \frac{2}{3^{i+1}}\right)=-\frac{40}{3^{2 i+4}}, \quad \mathcal{G}_{1}\left(0, \frac{2}{3^{i}}\right)=-\frac{176}{3^{2 i+4}} .
$$

we obtain from (B.6) and $\left(\right.$ B.7 that $d^{2}\left((x, z),\left(\frac{2}{3^{i+2}}, \frac{2}{3^{i+1}}\right)\right)<|(x, z)|^{2}$ for all $(x, z) \in T_{1, i}$, which in particular implies (B.5). 
We define:

$$
\ell:=\left\{(x, g(x)):|(x, g(x))-q| \leq|q|, x \geq x_{q}\right\}, \quad \text { for } g(x):=-\frac{z_{q}-\frac{2}{3^{i+1}}}{\frac{2}{3^{i+2}}-x_{q}}\left(x-x_{q}\right)+z_{q} .
$$

We have that $g$ is well-defined because of (B.4). In fact, $\ell$ is the segment with one end in $q$ and containing $\left(\frac{2}{3^{i+2}}, \frac{2}{3^{i+1}}\right)$ because of $(\mathrm{B} .5)$ and because $g\left(\frac{2}{3^{i+2}}\right)=\frac{2}{3^{i+1}}$. Moreover, we recall that:

$$
\left(\frac{2}{3^{i+2}}, \frac{2}{3^{i+1}}\right) \subset \partial \Omega \subset \mathbb{R}^{2} \backslash \Omega \text {. }
$$

In addition, using $\left(\mathrm{B} .2_{3}\right.$ and $(\mathrm{B} .4)$ we obtain that:

$$
\frac{\frac{2}{3^{i+1}}-z_{q}}{\frac{2}{3^{i+2}}-x_{q}}>\frac{\frac{2}{3^{i+1}}-\frac{2}{3^{i}}+6 x_{q}}{\frac{2}{3^{i+2}}-x_{q}}=-\frac{\frac{4}{3^{i+1}}-6 x_{q}}{\frac{2}{3^{i+2}}-x_{q}}=-6 .
$$

Considering that in a neighbourhood of $\left(\frac{2}{3^{i+2}}, \frac{2}{3^{i+1}}\right)$ the set $\partial \Omega$ is a segment of slope -6 (see (B.1) for $i+2$ instead of $i$ ) and (B.9), we get by continuity for some $\varepsilon_{q}>0$ small enough that:

$$
\left(\frac{2}{3^{i+2}}+\varepsilon_{q}, g\left(\frac{2}{3^{i+1}}+\varepsilon_{q}\right)\right) \in \ell \cap \Omega .
$$

Consequently, $\ell$ is a segment of length $|q|$, with one end in $q$ and such that $\ell \cap \Omega$ is not connected.

Case 3: if $z_{q} \geq 3 x_{q}$ and if there is $i \in \mathbb{N}_{*}$ such that $z_{q} \in\left[2 / 3^{i+1}, 2 / 3^{i}\right.$ ) and $z_{q} \geq \frac{2}{3^{i}}-6 x_{q}$ (see $q_{3}$ in Figure 5). Under these hypotheses $q$ is in the triangle:

$$
T_{2, i}:=\left\{(x, z): z<\frac{2}{3^{i}}, z \geq \frac{2}{3^{i}}-6 x, z \geq 3 x\right\} \backslash\left\{\left(\frac{2}{3^{i+1}}, \frac{2}{3^{i}}\right)\right\},
$$

whose vertices are given by:

$$
\left\{\left(0, \frac{2}{3^{i}}\right),\left(\frac{2}{3^{i+2}}, \frac{2}{3^{i+1}}\right),\left(\frac{2}{3^{i+1}}, \frac{2}{3^{i}}\right)\right\} .
$$

It can be deduced from $(\mathrm{B} .10)$ that:

$$
T_{2, i} \subset\left\{(x, z): z>\frac{2}{3^{i+1}}\right\} .
$$

We remark that the horizontal distance of the points in the line $z=\frac{2}{3^{i}}-6 x$ with respect to the line $z=\frac{2}{3^{i-1}}-6 x$ is given by $\frac{2}{3^{i+1}}$ (both lines are parallel). This implies that the vertical distance of $q$ to the line $z=\frac{2}{3^{i-1}}-6 x$ is bounded by $\frac{2}{3^{i+1}}$. Moreover, because of (B.11) we have that $|(x, z)|>\frac{2}{3^{i+1}}$ for all $(x, z) \in T_{2, i}$. Thus, the segment $\ell=\left\{\left(x, z_{q}\right): x \in\left[x_{q}, x_{q}+|q|\right]\right\}$ is of length $|q|$, one of its ends is $q$, and satisfies that $\ell \cap \Omega$ is not connected, as $\left(\frac{2}{3^{i+1}}, z_{q}\right) \in \ell \cap\left(\mathbb{R}^{2} \backslash \Omega\right)$ and $\left(\frac{1}{3^{i}}-\frac{z_{q}}{6}+\varepsilon_{q}, z_{q}\right) \in \ell \cap \Omega$ for some $\varepsilon_{q}>0$ small enough (see (B.1) for $i+1$ instead of $i$ ).

Remark B.4. The construction given in this section can clearly be generalized to higher dimensions. 


\section{References}

[1] R. A. Adams and J. J. F. Fournier. Sobolev spaces, volume 140. Elsevier, 2003.

[2] J. Apraiz and L. Escauriaza. Null-control and measurable sets*. ESAIM: COCV, 19(1):239$254,2013$.

[3] J. Apraiz, L. Escauriaza, G. Wang, and C. Zhang. Observability inequalities and measurable sets. J. Eur. Math. Soc, 16(11), 2014.

[4] A. Benabdallah, Y. Dermenjian, and J. Le Rousseau. Carleman estimates for the onedimensional heat equation with a discontinuous coefficient and applications to controllability and an inverse problem. J. Math. Anal. Appl., 336(2):865-887, 2007.

[5] F. Boyer and P. Fabrie. Mathematical tool for the study of the incompressible Navier-Stokes equations and related models. Springer, first edition, 2013.

[6] F. W. Chaves-Silva and G. Lebeau. Spectral inequality and optimal cost of controllability for the Stokes system. ESAIM: CoCV, 22(4):1137-1162, 2016.

[7] B. Csikós. Differential geometry. Typotex Publishing House, 2014.

[8] A. Doubova, E. Fernández-Cara, M. González-Burgos, and E. Zuazua. On the controllability of parabolic systems with a nonlinear term involving the state and the gradient. SIAM J. Control. and Optim., 41(3):798-819, 2002.

[9] A. Doubova, A. Osses, and J.-P. Puel. Exact controllability to trajectories for semilinear heat equations with discontinuous diffusion coefficients. ESAIM: COCV, 8:621-661, 2002.

[10] S. Ervedoza and E. Zuazua. Sharp observability estimates for heat equations. Arch. Ration. Mech. An., 202(3):975-1017, 2011.

[11] L. Escauriaza, S. Montaner, and C. Zhang. Observation from measurable sets for parabolic analytic evolutions and applications. J. Math. Pure. Appl., 104(5):837-867, 2015.

[12] L. Escauriaza, S. Montaner, and C. Zhang. Analyticity of solutions to parabolic evolutions and applications. SIAM J. Control. and Optim., 49(5):4064-4092, 2017.

[13] C. Fabre, J.-P. Puel, and E. Zuazua. Approximate controllability of the semilinear heat equation. Proc. R. Soc. E. A.-Ma., 125(1):31-61, 1995.

[14] E. Fernández-Cara. Null controllability of the semilinear heat equation. ESAIM: COCV, 2:87-103, 1997. 
[15] E. Fernández-Cara, M. González-Burgos, S. Guerrero, and J.-P. Puel. Null controllability of the heat equation with boundary Fourier conditions: the linear case. ESAIM: COCV, $12(3): 442-465,2006$.

[16] E. Fernández-Cara and S. Guerrero. Global Carleman estimates for solutions of parabolic systems defined by transposition and some applications to controllability. Appl. Math. Res. Express, 2006.

[17] E. Fernández-Cara and S. Guerrero. Global carleman inequalities for parabolic systems and applications to controllability. SIAM J. Control. and Optim., 45(4):1395-1446, 2006.

[18] E. Fernández-Cara and E. Zuazua. Null and approximate controllability for weakly blowing up semilinear heat equations. In Ann. I. H. Poincare-A.N., volume 17, pages 583-616. Elsevier, 2000.

[19] A. V. Fursikov and O. Yu. Imanuvilov. Controllability of evolution equations. Number 34. Seoul National University, 1996.

[20] M. González-Burgos and L. de Teresa. Some results on controllability for linear and nonlinear heat equations in unbounded domains. Adv. Differential Equ., 12(11):1201-1240, 2007.

[21] P. Grisvard. Elliptic problems in nonsmooth domains. SIAM, 2011.

[22] S. Guerrero and F. Guillen-Gonzalez. On the controllability of the hydrostatic stokes equations. J. Math. Fluid Mech., 10(3):402-422, 2008.

[23] O. Yu. Imanuvilov, J.-P. Puel, and M. Yamamoto. Carleman estimates for parabolic equations with nonhomogeneous boundary conditions. Chin. Ann. Math., 30(4):333-378, 2009.

[24] O. Yu. Imanuvilov and M. Yamamoto. Carleman estimate for a parabolic equation in a Sobolev space of negative order and its applications. In Control of Nonlinear Distributed Parameter Systems, pages 137-162. CRC Press, 2001.

[25] D. S. Jerison and C. E. Kenig. The Neumann problem on Lipschitz domains. B. Am. Math. Soc., 4(2):203-207, 1981.

[26] J. Le Rousseau and L. Robbiano. Local and global Carleman estimates for parabolic operators with coefficients with jumps at interfaces. Invent. Math., 183(2):245-336, 2011.

[27] G. Lebeau and L. Robbiano. Contrôle exact de l'équation de la chaleur. Commun. Part. Diff. Eq., 20(1-2):335-356, 1995. 
[28] G. Lieberman. Regularized distance and its applications. Pac. J. Math., 117(2):329-352, 1985 .

[29] J. L. Lions. Contrôlabilité exacte, perturbations et stabilisation de systemes distribués, tome 1, RMA 8, 1988.

[30] J. L. Lions and E. Magenes. Problèmes aux limites non homogènes et applications. 1968.

[31] L. Miller. Geometric bounds on the growth rate of null-controllability cost for the heat equation in small time. J. Differ. Equations, 204(1):202-226, 2004.

[32] L. Miller. The control transmutation method and the cost of fast controls. SIAM J. Control. and Optim., 45(2):762-772, 2006.

[33] J. Pedlosky. Geophysical fluid dynamics. Springer-Verlag, 1982.

[34] D. L. Russell. A unified boundary controllability theory for hyperbolic and parabolic partial differential equations. Stud. Appl. Math., 52(3):189-211, 1973.

[35] D. L. Russell. Controllability and stabilizability theory for linear partial differential equations: recent progress and open questions. Siam Rev., 20(4):639-739, 1978.

[36] J. Simon. Compact sets in the space $L^{p}(O, T ; B)$. Ann. Mat. Pur. ed Appl., 146(1):65-96, 1986.

[37] G. Tenenbaum and M. Tucsnak. New blow-up rates for fast controls of Schrödinger and heat equations. J. Differ. Equations, 243(1):70-100, 2007.

[38] G. Wang. $L^{\infty}$-null controllability for the heat equation and its consequences for the time optimal control problem. SIAM J. Control. and Optim., 47(4):1701-1720, 2008.

[39] C. Zhang. An observability estimate for the heat equation from a product of two measurable sets. J. Math. Anal. Appl., 396(1):7-12, 2012. 